\title{
Selectively Targeting Tumor Hypoxia with the Hypoxia-Activated Prodrug CP-506
}

\section{Alexander M.A. van der Wiel}

The University of Maastricht

\section{Victoria Jackson-Patel}

The University of Auckland

\section{Raymon Niemans}

The University of Maastricht

\section{Ala Yaromina}

The University of Maastricht

\section{Emily Liu}

The University of Auckland

\section{Damiënne Marcus}

The University of Maastricht

\section{Alexandra Mowday}

The University of Maastricht

\section{Natasja Lieuwes}

The University of Maastricht

\section{Rianne Biemans}

The University of Maastricht

\section{Xiaojing Lin}

The University of Auckland

\section{Zhe Fu}

The University of Auckland

\section{Sisira Kumara}

The University of Auckland

\section{Arthur Jochems}

The University of Maastricht

\section{Amir Ashoorzadeh}

The University of Auckland

\section{Robert Anderson}

The University of Auckland

\section{Kevin Hicks}

The University of Auckland 
The University of Auckland

\section{Maria Abbattista}

The University of Auckland

\section{Christopher Guise}

The University of Auckland

\section{Sofie Deschoemaeker}

Convert Pharma

\section{Sophie Thiolloy}

Convert Pharma

\section{Arne Heyerick}

Convert Pharma

\section{Morwena Solivio}

University of Minnesota

\section{Silvia Balbo}

University of Minnesota

\section{Jeff Smaill}

The University of Auckland

\section{Jan Theys}

The University of Maastricht

\section{Ludwig Dubois}

The University of Maastricht

Adam Patterson ( $\nabla$ a.patterson@auckland.ac.nz )

The University of Auckland https://orcid.org/0000-0001-5138-1227

\section{Philippe Lambin}

The University of Maastricht https://orcid.org/0000-0001-7961-0191

\section{Research Article}

Keywords: Hypoxia, Cancer, Hypoxia-Activated Prodrugs, Precision Medicine, Biomarker

Posted Date: April 19th, 2021

DOl: https://doi.org/10.21203/rs.3.rs-438613/v1

License: (c) (i) This work is licensed under a Creative Commons Attribution 4.0 International License. Read Full License 


\section{Abstract \\ Background}

Hypoxia-activated prodrugs (HAPs) are a promising class of antineoplastic agents that can selectively eliminate hypoxic tumor cells. The present study evaluates the hypoxia-selectivity and antitumor activity of CP-506, a DNA alkylating HAP with favorable pharmacological properties.

\section{Methods}

Stoichiometry of reduction, one-electron affinity, and back-oxidation rate of CP-506 were characterized by fast-reaction radiolytic methods. In vitro, 2D monolayer and 3D spheroid and multicellular layer cultures were used to investigate the hypoxia-selectivity of CP-506. In vivo, the causal relationship between tumor oxygenation and antitumor effects of CP-506 was assessed. Mice bearing a range of human tumor xenografts were exposed to CP-506 and tumor growth was monitored. A multivariate linear regression model was used to identify factors associated with CP-506 treatment outcome.

\section{Results}

Net reduction, metabolism, and cytotoxicity of CP-506 were maximally inhibited at oxygen concentrations above $1 \mu \mathrm{M}\left(0.1 \% \mathrm{O}_{2}\right)$. CP-506 demonstrated cytotoxicity selectively in hypoxic 2D and 3D cell cultures with normoxic/anoxic $\mathrm{IC}_{50}$ ratios up to 203. In vivo, the antitumor effects of $\mathrm{CP}-506$ were selective for hypoxic tumor cells and causally related to tumor oxygenation. CP-506 effectively decreased the hypoxic fraction and inhibited growth of a wide range of hypoxic xenografts. Two well-oxygenated models were refractory to treatment despite intrinsic anoxic sensitivity in vitro. A multivariate regression analysis revealed baseline tumor hypoxia and in vitro sensitivity to CP-506 to significantly correlate with treatment response.

\section{Conclusions}

Our results demonstrate that CP-506 selectively sterilizes hypoxic tumor cells and has broad antitumor activity. Our data also indicate that tumor hypoxia and cellular sensitivity to CP-506 are strong determinants of the antitumor effects of CP-506.

\section{Background}

Hypoxia is a common feature in the majority of solid tumors that arises through disruption of the balance between proliferation and oxygen supply (1). Given its pivotal role in tumor progression and resistance to conventional therapies (2), several strategies have been developed to overcome tumor hypoxia, including the use of hypoxia-activated prodrugs (HAPs) (3). HAPs are low-molecular weight therapeutic agents that, 
only under sufficiently low oxygen tension, are bioactivated by enzymatic reduction to typically generate cytotoxic effector molecules (4).

Six HAPs have previously been evaluated in a clinical setting $(5,6)$. Evofosfamide (TH-302), a 2nitroimidazole-based phosphoramidate mustard HAP, demonstrated single-agent antitumor activity (7) that is further increased when combined with conventional anticancer therapies, both preclinically (8-10) and clinically $(11,12)$. However, addition of evofosfamide to standard of care treatment failed to improve overall survival in pancreatic cancer (NCT01746979) (13) and soft tissue carcinoma (NCT01440088) (14). Potential explanations are a lack of patient stratification (3), unexpected lowering of pharmacokinetic exposures due to prodrug reformulation during the Phase III trials (15), and limited diffusion of its cytotoxic metabolite (bystander effect) $(16,17)$. PR-104, a phosphate pre-prodrug of the dinitrobenzamide nitrogen mustard HAP PR-104A, demonstrated hypoxia-selective activation in vitro (PR$104 \mathrm{~A}$ ) and in vivo (PR-104) (18). The combination of PR-104 with radio- or chemotherapy further enhanced its antitumor effects (18-20). However, clinical safety and tolerability of PR-104 demonstrated dose-limiting thrombocytopenia and neutropenia $(21,22)$. Subsequent preclinical studies elucidated that PR-104A can be activated independent of tissue oxygenation by human two-electron aldo-keto reductase $1 C 3$ (AKR1C3) $(23,24)$. Further clinical evaluation revealed that myelotoxicity was the only serious adverse event preventing dose-escalation of PR-104 administration (25) and reaching adequate plasma concentrations for monotherapeutic efficacy $(21,22,26)$. Hence, avoiding AKR1C3 bioactivation should suppress prodrug myelotoxicity and permit significant dose-escalation without encountering treatmentrelated hematological adverse effects.

Based on this rationale, PR-104 was redesigned to give rise to CP-506, a next-generation HAP with more favorable properties (27-29). Firstly, CP-506 is designed to be resistant to AKR1C3 metabolism, ensuring highly specific hypoxia-dependent activation. Secondly, CP-506 is a water-soluble piperazine mesylate salt, avoiding the need for a phosphate solubilization strategy utilized for PR-104, and is thus resistant to glucuronidation of the alcohol present in PR-104A, a major clearance pathway compromising its pharmacokinetic profile $(30,31)$. Thirdly, CP-506 mesylate has the potential to be orally bioavailable. Fourthly, CP-506 is a mono-nitro HAP, thereby avoiding facile metabolic loss via self-alkylation of the reduced nitro-group ortho to the mustard (18). Finally, physiochemical properties of CP-506 and its metabolites readily permit a bystander effect. The proposed mechanism of action of CP-506 (Fig. 1A) is similar to that of PR-104 (Supplementary Fig. 1).

In the present study, we first characterized the stoichiometry of CP-506 reduction, one-electron affinity, and back-oxidation rate by using fast-reaction radiolytic methods. We accurately assessed the oxygendependence of CP-506 metabolism and cytotoxicity and evaluated resistance to aerobic AKR1C3 metabolism and the hypoxia-selective cytotoxicity and DNA adduct formation of CP-506 in a wide range of 2D and 3D in vitro models. In vivo, we first assessed whether a causal relationship existed between CP506 efficacy and tumor oxygenation. We further characterized the antitumor effects of CP-506 in a broad panel of 15 human xenograft models. We hypothesized that CP-506 is effective in inhibiting growth of 
hypoxic tumors only, reducing their hypoxic fraction. Lastly, a multivariate linear regression analysis was performed to identify which factors ultimately contribute to CP-506 antitumor efficacy.

\section{Methods}

\section{Compounds}

CP-506 (2-[(2-bromoethyl)-5-[(4-ethyl-1-piperazinyl)carbonyl]-2-(methylsulfonyl)-4-nitroanilino]ethyl methanesulfonate), $\mathrm{CP}-506 \mathrm{H}$ (2-((2-bromoethyl)(5-(4-ethylpiperazine-1-carbonyl)-4-(hydroxyamino)-2(methylsulfonyl)phenyl)amino)ethyl methanesulfonate), and CP-506M (2-((4-amino-5-(4-ethylpiperazine1-carbonyl)-2-(methylsulfonyl)phenyl)(2-bromoethyl)amino)ethyl methanesulfonate) were manufactured by Mercachem employing synthetic routes developed at the University of Auckland (Supplementary Materials and Methods). Deuterated internal standards were prepared using identical synthetic procedures employing D8-1-ethylpiperazine instead of 1-ethylpiperazine. SN35393 (5-(bis(2bromoethyl)amino)-4-(methylsulfonyl)-2-nitrophenyl)(4-methylpiperazin-1-yl)methanone) was synthesized as described previously (27). PR-104 was supplied by Proacta Inc. PR-104A was synthesized and purified as previously reported $(32,33)$. For in vitro experiments, stock solutions were made in dimethyl sulfoxide (DMSO) and stored at $-20^{\circ} \mathrm{C}$. For in vivo experiments, compounds were dissolved in water for injection (WFI).

\section{Pulse and steady-state radiolysis studies}

The one-electron reduction potential of CP-506 $\left(E^{0^{\prime}}\left(\mathrm{S} / \mathrm{S}^{-}{ }^{-}\right)\right)$was determined by a previously described pulse radiolysis method (34). UV-visible spectrum of the radical anion was produced by directly scavenging the radiolytically produced $\mathrm{e}^{-}$aq and by electron transfer from the $\mathrm{CO}_{2} \cdot{ }^{-}$radical. The backoxidation rates of the radical anion were followed by time-resolved spectrophotometry with increasing concentrations of dissolved oxygen from which the second-order rate constant was determined. The stepwise reduction of CP-506 was followed using spectrophotometry by the addition of reducing equivalents in anoxia produced upon steady-state radiolysis using a y-ray ${ }^{137} \mathrm{Cs}$ source.

\section{Cell culture}

Cells were routinely cultured at $37^{\circ} \mathrm{C}$ in a humidified $5 \% \mathrm{CO}_{2}$ air atmosphere and were STR-authenticated and confirmed to be mycoplasma-free by PCR (Roche Diagnostics). Tissue of origin, provider, and culture medium of the employed cell lines are summarized in Supplementary Table 1. HCT116 cells overexpressing the human aldo-keto reductases $1 \mathrm{C}$ family members and human diflavin reductases were previously described $(23,35)$.

\section{Assessment of oxygen-dependence of CP-506 activation}

Stirred suspensions of HCT116 cells overexpressing cytochrome P450 oxidoreductase (POR; HCT116 ${ }^{\mathrm{POR}}$ ) were exposed to variable gas mixtures $\left(0-95 \% \mathrm{O}_{2}, 5 \% \mathrm{CO}_{2}\right.$, residual $\left.\mathrm{N}_{2}\right)$. After pre-equilibration, cell suspension were treated with CP-506 (100 $\mu \mathrm{M}, 1 \mathrm{~h})$. Soluble (aqueous) oxygen concentrations were 
measured directly using a calibrated FOSPOR fiber-optic oxygen probe (Oxygen Optics). For metabolic endpoint, samples were crashed in acetonitrile containing deuterated internal standards and stored $(-80 \otimes C)$ until LC-MS/MS analysis. For cell survival endpoint, samples were harvested and clonogenic survival was assessed after 12 days. Colonies ( $>50$ cells) were manually counted to determine plating efficiency (PE). A linear regression between $\mathrm{CP}-506 \mathrm{H}$ formation or PE and CP-506 AUC was fitted and the $\mathrm{KO}_{2}$ value, defined as the $\mathrm{O}_{2}$ concentration required to reduce the effect of CP-506 to $50 \%$ of that effect in anoxic culture, was assessed.

\section{Cell viability assays}

Culture medium, 96-well plates, and pipet tips were preincubated $72 \mathrm{~h}$ before use in normoxic or anoxic conditions in respectively a cell culture incubator (HERAcell® $150 \mathrm{CO}_{2}$ Incubator; $21 \% \mathrm{O}_{2}, 5 \% \mathrm{CO}_{2}$ ) or anoxic workstations (A35 Don Whitley, Don Whitley Scientific; Bactron II, Shell Lab; $<1$ ppm $\mathrm{O}_{2}, 10 \% \mathrm{H}_{2}$, $5 \% \mathrm{CO}_{2}$, residual $\mathrm{N}_{2}$ ). Cells were transferred to normoxic or anoxic conditions and resuspended in preincubated medium. Cells were seeded in 96-well plates in optimized densities and were allowed to attach for $2 \mathrm{~h}$. CP-506 was dissolved in preincubated medium and cells were exposed to CP-506containing medium for $4 \mathrm{~h}$. After, plates were transferred to normoxia, washed, and received fresh culture medium. Cell viability was assessed $96 \mathrm{~h}$ after start of treatment using CellTiter-Glo® 2.0 reagent (Promega) or Sulforhodamine B (SRB) assay (Sigma-Aldrich) according to the manufacturer's protocol. Treatment response was quantified as $\mathrm{IC}_{50}$, i.e. the concentration of $\mathrm{CP}-506$ that reduced cell viability by $50 \%$. The hypoxia cytotoxicity ratio (HCR) was defined as the ratio of the normoxic to anoxic $\mathrm{IC}_{50}$.

\section{Multicellular layer clonogenic cell survival assay}

Multicellular layers (MCL) were grown as described previously (36). MCLs were exposed to CP-506 for $5 \mathrm{~h}$ under anoxic or hyperoxic $\left(95 \% \mathrm{O}_{2}, 5 \% \mathrm{CO}_{2}\right)$ conditions. MCLs were trypsinized and clonogenic survival was assessed after 10 days. Treatment response was quantified as $\mathrm{IC}_{10}$, i.e. the concentration of CP-506 resulting in $90 \%$ clonogenic cell kill.

\section{Adductomics analyses}

The LC-MS approach used for the identification and relative quantification of CP-506-induced DNA adduct formation is described in Supplementary Materials and Methods.

\section{Spheroid culture}

Spheroids were grown as described previously (37). Monitoring of spheroid growth and assessment of oxygenation status is described in Supplementary Materials and Methods. To assess the effect of CP-506 on spheroid growth, normoxic and hypoxic spheroids were treated with CP-506 for $4 \mathrm{~h}$, after which spheroids received fresh culture medium and growth was monitored.

\section{Animals}

All animal experiments were performed with appropriate ethical approval and according to institutional guidelines. Additional information on the animal experiments performed in this study are described in 


\section{Pharmacokinetic studies}

Pharmacokinetic properties of CP-506 were monitored in NIH-III mice following intravenous (IV), oral (PO), or intraperitoneal (IP) administration at indicated time points. Sample collection, LC/MS analysis, and assessment of pharmacokinetic parameters are described in Supplementary Materials and Methods.

\section{Tumor models and treatment schedules}

To generate tumors, cells were resuspended in 50\% Matrige $\mathrm{I}^{\mathrm{TM}}$ (BD Biosciences) or aMEM and injected subcutaneously into the flank of the animal. Mice were randomly assigned to treatment groups upon a tumor volume of $c a .200 \mathrm{~mm}^{3}$ (treatment starting volume, SV). To evaluate the antitumor effects of CP506 , mice received different dose-regimens of vehicle or CP-506. To investigate the causal relationship between the antitumor effects of CP-506 and tumor oxygenation, mice bearing MDA-468 xenografts were exposed to $7 \% \mathrm{O}_{2}, 21 \% \mathrm{O}_{2}$, or carbogen $\left(95 \% \mathrm{O}_{2}, 5 \% \mathrm{CO}_{2}\right)$ and nicotinamide $(500 \mathrm{mg} / \mathrm{kg}$, IP; Sigma Aldrich) (CarbN) in controlled breathing chambers for a total of 2.5 h. 30 min after start of modified breathing conditions, vehicle or CP-506 (600 mg/kg, IP, QD1) were injected. Tumor response was quantified as (I) tumor growth inhibition (TGI) at the day respective control animals reached four times SV (4xSV), (II) as tumor growth delay (TGD) defined as time to reach 4xSV (T4xSV), or (III) as specific growth delay (SGD). To estimate and compare the magnitude of treatment effect, the enhancement ratio (ER), defined as the ratio of T4xSV of CP-506-treated animals to T4xSV of vehicle-treated animals, was determined. Assessment of tumor volumes and definition of TGI and SGD are described in Supplementary Materials and Methods.

\section{Tumor excision assay}

H460 xenograft-bearing mice were exposed to sham or whole body irradiation (10 Gy), followed by administration of vehicle or CP-506 (900 or $1100 \mathrm{mg} / \mathrm{kg}$, IP, QD1). $18 \mathrm{~h}$ after, tumors were excised, homogenized, and plated. Clonogenic survival was assessed after 10 days. Log cell kill (LCK) relative to sham-treated controls were calculated.

\section{Assessment of tumor hypoxia and DNA damage}

Mice bearing vehicle- or CP-506-treated xenografts were injected with the hypoxia markers pimonidazole ( $60 \mathrm{mg} / \mathrm{kg}$, IP in saline), EF5 (30 mg/kg, IP in saline; Sigma Aldrich) respectively $1 \mathrm{~h}$ and $3 \mathrm{~h}$ before tumor harvesting, and/or CCl-103F (100 mg/kg, IP in 10\% (v/v) DMSO in peanut oil; NPI, Inc.) $2 \mathrm{~h}$ before the start of treatment depending on the experiment. Subsequent collection and staining of tumors, image acquisition and analysis are described in Supplementary Materials and Methods.

\section{Statistics}

Statistical analyses were performed in GraphPad Prism 8.4.3 software (GraphPad Software, Inc.) or SigmaStat v14.1 (Systat Software). Differences in T4xSV and HF were analyzed using an unpaired t-test or one-way ANOVA. Differences in survival were analyzed using a log-rank test. P values $\leq 0.05$ were 
considered statistically significant. A multivariate linear regression analysis was performed (STATA/IC 11.1) on a dataset comprising data of 381 animals to test which parameters significantly influenced ER or SGD. Details of this analysis are described in Supplementary Materials and Methods. A nomogram predicting ER was subsequently constructed in R (v4.0.2).

\section{Results}

\section{Reduction of CP-506 to its radical anion is inhibited by oxygen}

The one-electron reduction potential $\left(E^{0}\right)$ of $\mathrm{CP}-506$ at $\mathrm{pH} 7$ vs. normal hydrogen electrode was $-351 \pm 8$ $\mathrm{mV}$ (Fig. 1A, Supplementary Fig. 2A), within the appropriate range to undergo one-electron reduction by endogenous oxidoreductases. The second-order back-oxidation rate constant of the radical anion by $\mathrm{O}_{2}$ was determined as $2.45 \pm 0.08 \times 10^{6} \mathrm{M}^{-1} \mathrm{~s}^{-1}$, conformant with other nitroarene prodrugs of similar $E^{O^{\prime}}$ (Supplementary Fig. 2B). Stepwise reduction of CP-506 upon steady-state radiolysis under anoxia resulted in spectral changes with maintenance of isosbestic points and complete reduction of the compound at $\mathrm{G}$ (loss) $=1.01 \pm 0.04 \times 10^{-7}{\mathrm{M} . G{ }^{-1}}^{-1}$, requiring six reducing equivalents (Supplementary Fig. $2 \mathrm{C}$ and $2 \mathrm{D}$ ). These data indicate a complete six-electron reduction of the nitro substituent to the amine (CP-506M) and identify it as a probable cytotoxic species formed under anoxic conditions.

\section{Metabolism and cytotoxicity of CP-506 is inhibited by trace oxygen}

To evaluate the $\mathrm{O}_{2}$-dependence of metabolism and cytotoxicity of CP-506, HCT116 ${ }^{\mathrm{POR}}$ single-cell stirred suspensions were exposed to CP-506 under increasing solution-phase $\mathrm{O}_{2}$ concentrations. CP-506 concentration declined as function of aqueous soluble $\mathrm{O}_{2}$ with maximal metabolic consumption achieved under anoxia. Metabolic consumption of CP-506 was accompanied by formation of CP-506H and CP$506 \mathrm{M}$. Data was fitted for $\mathrm{CP}-506 \mathrm{H}$ formation with a four-parameter sigmoidal curve $\left(\mathrm{R}^{2}=0.703\right)$, suggesting a $\mathrm{KO}_{2}$ value of $0.57 \mu \mathrm{M} \mathrm{O}_{2}$ with metabolism completely inhibited by soluble $\mathrm{O}_{2}$ concentrations above $1 \mu \mathrm{M}$ (Fig. 1B). Consistent with the hypoxia-selective metabolism, the cytotoxicity of CP-506 was maximal in anoxic cultures and was progressively inhibited by increasing solution-phase $\mathrm{O}_{2}$. The slope of the k-curve $\left(R^{2}=0.504\right)$ suggests a $\mathrm{KO}_{2}$ value of $0.60 \mu \mathrm{M} \mathrm{O}_{2}$, with clonogenic killing completely inhibited by $\mathrm{O}_{2}$ concentrations above $1 \mu \mathrm{M}$ (Supplementary Fig. 2E). These data indicate that metabolism and cytotoxicity of CP-506 are readily inhibited by trace $\mathrm{O}_{2}$.

\section{CP-506 is resistant to aerobic activation and is selectively cytotoxic in hypoxic 2D cell cultures}

First, to validate that CP-506 is, unlike PR-104A, resistant to aerobic activation by the human two-electron reductase AKR1C3, HCT116 parental (WT) and HCT116 cells overexpressing the AKR1C family members 
(AKR1C1 - AKR1C4) were exposed to each prodrug under normoxic conditions. HCT116 AKR1C3 cells displayed 112-fold hypersensitivity to PR-104A relative to HCT116 ${ }^{W T}$ cells $(p<0.01)$, whilst the remaining AKR1C-expressing cell lines were refractory (Fig. 1C). In contrast, CP-506 failed to inhibit the proliferation of the AKR1C expressing cells, including HCT116 ${ }^{\text {AKR1C3 }}$ cells. To account for potential differences in metabolite loss, HCT116 WT and HCT116 ${ }^{\mathrm{AKR} 1 \mathrm{C} 3}$ 3D MCLs were exposed to $10 \mu \mathrm{M}$ PR-104A or CP-506 under hyperoxic conditions. Exposure to PR-104A drastically reduced (>99.9\%) clonogenic survival of HCT116 ${ }^{\text {AKR1C3 }}$ but not of HCT116 ${ }^{\text {WT }}$ MCLs, whereas no effect was observed upon CP-506 treatment (Supplementary Fig. 3A). To confirm these findings in vivo, isogenic SNU-398 tumors, either AKR1C3negative parental (WT) or overexpressing AKR1C3 were treated with equimolar doses $(950 \mu \mathrm{mol} / \mathrm{kg})$ of PR-104 or CP-506. SNU-398 ${ }^{\mathrm{WT}}$ tumors were refractory to either treatment (Supplementary Fig. 3B), whereas SNU-398 ${ }^{\text {AKR1C3 }}$ tumors rapidly regressed upon PR-104, but not CP-506 treatment (Fig. 1D). Similar results were observed in HCT116 isogenic tumor models (Supplementary Fig. 3C and 3D). Taken together, these data confirm that CP-506 is resistant to AKR1C3-mediated aerobic bioactivation.

Next, to determine whether CP-506 is a substrate for endogenous human one-electron oxidoreductases, HCT116 cells overexpressing human diflavin reductases were exposed to CP-506 under normoxia or anoxia. Relative to parental cells, anoxic $\mathrm{IC}_{50}$ values of CP-506 were significantly $(p \leq 0.01)$ lower in HCT116 cells overexpressing POR, MTRR, NDOR1, and NOS2A, but not NOS3, whereas no differences in normoxic $\mathrm{IC}_{50}$ values were observed (Fig. 1E). To confirm that CP-506 retains hypoxia-selective cytotoxicity in vitro, we assessed normoxic and anoxic $\mathrm{IC}_{50}$ values and corresponding HCRs of CP-506 and PR-104A in MDA-468, C33A, and SiHa (Supplementary Figs. 3E-G). CP-506 was consistently more cytotoxic under anoxia (HCR: 203, 55, and 20, respectively), whereas PR-104A displayed less hypoxiaselectivity (HCR: 65, 23, and 7, respectively). Lastly, IC ${ }_{50}$ values of CP-506 were determined in a broad panel of human tumor cell lines (Table 1). For all cell lines, normoxic $\mathrm{IC}_{50}$ values were higher than anoxic $\mathrm{IC}_{50}$ values, resulting in HCRs ranging from 4 to 157 . These data demonstrate the hypoxia-selective bioactivation and cytotoxicity of CP-506 in in vitro monolayer cultures. 
Table 1

IC50 values in normoxic $(21 \% 02)$ and anoxic $(\leq 0.02 \% 02)$ in vitro monolayers after $4 \mathrm{~h}$ exposure to CP506.

\begin{tabular}{|c|c|c|c|c|}
\hline Cell line & Cancer type & $\mathrm{IC}_{50}$ normoxia $(\mu \mathrm{M})$ & $\mathrm{IC}_{50}$ anoxia $(\mu \mathrm{M})$ & HCR \\
\hline MDA-453 & Breast adenocarcinoma & 202 & 1.5 & 139 \\
\hline SW1990 & Pancreatic adenocarcinoma & 133 & 1.7 & 78.8 \\
\hline DMS 114 & Small cell lung carcinoma & 360 & 3.6 & 100 \\
\hline MiaPaCa-2 & Pancreatic ductal adenocarcinoma & 646 & 4.1 & 157 \\
\hline MDA-468 & Breast adenocarcinoma & 243 & 4.7 & 51.6 \\
\hline$A-427$ & Lung adenocarcinoma & 374 & 9.3 & 40.3 \\
\hline Capan-1 & Pancreatic ductal adenocarcinoma & 136 & 10.7 & 12.8 \\
\hline EVSA-T & Breast carcinoma & 401 & 15.8 & 25.3 \\
\hline MDA-436 & Breast adenocarcinoma & 178 & 17.5 & 10.2 \\
\hline T47D & Breast ductal adenocarcinoma & 671 & 18.3 & 36.7 \\
\hline H460 & Large cell lung carcinoma & 319 & 18.4 & 17.4 \\
\hline SK-BR-3 & Breast carcinoma & 403 & 21.7 & 18.6 \\
\hline AsPC-1 & Pancreatic ductal adenocarcinoma & 368 & 21.9 & 16.8 \\
\hline LCLC-103H & Lung adenocarcinoma & 646 & 21.9 & 29.4 \\
\hline H1975 & Non-small cell lung carcinoma & 1003 & 26.2 & 38.3 \\
\hline BT-474 & Breast ductal adenocarcinoma & 1500 & 27.0 & 55.5 \\
\hline CAL-51 & Breast adenocarcinoma & 355 & 27.8 & 12.7 \\
\hline HCC-1143 & Breast ductal adenocarcinoma & 1340 & 28.0 & 47.9 \\
\hline EFM-192A & Breast adenocarcinoma & 1270 & 29.5 & 43.0 \\
\hline HCC-15 & Lung squamous cell carcinoma & 1246 & 29.8 & 41.9 \\
\hline EPLC-272H & Lung carcinoma & 857 & 34.3 & 25.0 \\
\hline JIMT-1 & Breast ductal adenocarcinoma & 875 & 35.0 & 25.0 \\
\hline CFPAC- 1 & Pancreatic ductal adenocarcinoma & 531 & 35.0 & 15.2 \\
\hline H1299 & Large cell lung carcinoma & 505 & 37.1 & 13.6 \\
\hline
\end{tabular}

$\mathrm{IC}_{50}$ values were determined by assessing cell viability $96 \mathrm{~h}$ after the start of treatment. The hypoxia cytotoxicity ratio $(\mathrm{HCR})$ was obtained by dividing the normoxic $\mathrm{IC}_{50}$ by the corresponding anoxic $\mathrm{IC}_{50}$. 


\begin{tabular}{|c|c|c|c|c|}
\hline Cell line & Cancer type & $\mathrm{IC}_{50}$ normoxia $(\mu \mathrm{M})$ & $\mathrm{IC}_{50}$ anoxia $(\mu \mathrm{M})$ & HCR \\
\hline A549 & Lung adenocarcinoma & 652 & 37.5 & 17.4 \\
\hline H1650 & Lung adenocarcinoma & 835 & 40.0 & 20.9 \\
\hline HPAF-II & Pancreatic ductal adenocarcinoma & 719 & 40.1 & 18.0 \\
\hline SU.86.86 & Pancreatic adenocarcinoma & 507 & 40.7 & 12.5 \\
\hline DAN-G & Pancreatic adenocarcinoma & 675 & 40.9 & 16.5 \\
\hline YAPC & Pancreatic carcinoma & 692 & 41.9 & 16.5 \\
\hline HCC1954 & Breast ductal adenocarcinoma & 987 & 42.9 & 23.0 \\
\hline HCC-827 & Lung adenocarcinoma & 581 & 43.1 & 13.5 \\
\hline SW1271 & Small cell lung carcinoma & 674 & 43.5 & 15.5 \\
\hline BT-549 & Breast ductal adenocarcinoma & 559 & 43.8 & 12.8 \\
\hline HUP-T4 & Pancreatic adenocarcinoma & 864 & 46.9 & 18.4 \\
\hline HDQ-P1 & Breast ductal adenocarcinoma & 1307 & 47.1 & 27.7 \\
\hline BxPC-3 & Pancreatic ductal adenocarcinoma & 1031 & 56.6 & 18.2 \\
\hline Panc-1 & Pancreatic ductal adenocarcinoma & 464 & 59.1 & 7.9 \\
\hline Capan-2 & Pancreatic ductal adenocarcinoma & 580 & 60.8 & 9.5 \\
\hline HCC1937 & Breast ductal adenocarcinoma & 808 & 70.6 & 11.5 \\
\hline CAL-120 & Breast adenocarcinoma & 1079 & 70.8 & 15.2 \\
\hline EFM-19 & Breast ductal adenocarcinoma & 512 & 83.2 & 6.2 \\
\hline CAL-12T & Non-small cell lung carcinoma & 833 & 84.9 & 9.8 \\
\hline HS-578T & Breast ductal adenocarcinoma & 1143 & 88.1 & 13.0 \\
\hline HCC-44 & Large cell lung carcinoma & 585 & 91.6 & 6.4 \\
\hline MDA-231 & Breast adenocarcinoma & 663 & 92.9 & 7.1 \\
\hline H520 & Lung squamous cell carcinoma & 1385 & 95.7 & 14.5 \\
\hline DMS 53 & Small cell lung carcinoma & 528 & 112 & 4.7 \\
\hline Hs766t & Pancreatic adenocarcinoma & 1268 & 154 & 8.2 \\
\hline BEN & Non-small cell lung carcinoma & 881 & 180 & 4.9 \\
\hline
\end{tabular}

$\mathrm{IC}_{50}$ values were determined by assessing cell viability $96 \mathrm{~h}$ after the start of treatment. The hypoxia cytotoxicity ratio $(\mathrm{HCR})$ was obtained by dividing the normoxic $\mathrm{IC}_{50}$ by the corresponding anoxic $\mathrm{IC}_{50}$. 


\begin{tabular}{|lllll|}
\hline Cell line & Cancer type & IC $_{50}$ normoxia $(\mu \mathrm{M})$ & IC $_{50}$ anoxia $(\mu \mathrm{M})$ & HCR \\
\hline $\mathrm{H} 1573$ & Lung adenocarcinoma & 1490 & 362 & 4.1 \\
\hline $\begin{array}{l}\mathrm{IC}_{50} \text { values were determined by assessing cell viability } \\
\text { cytotoxicity ratio }(\mathrm{HCR}) \text { was obtained by dividing the }\end{array}$ normoxic $\mathrm{IC}_{50}$ by the corresponding anoxic $\mathrm{IC}_{50}$. \\
\hline
\end{tabular}

\section{CP-506-DNA adducts specifically form under hypoxic conditions in vitro}

To demonstrate hypoxia-selective CP-506-DNA adduct formation, a targeted LC-MS approach focusing on 39 adducts identified earlier was employed. Exposure of cells to CP-506 under normoxic conditions resulted in formation of 2 or 3 DNA monoadducts. In contrast, cells exposed to CP-506 under anoxic conditions demonstrated formation of 9 to 15 DNA adducts, including several crosslinks (Supplementary Table 3). The majority of these adducts were formed due to the activated metabolite CP-506M, followed by $\mathrm{CP}-506 \mathrm{H}$. Collectively, these data support the hypoxia-specific activation of CP-506 and subsequent DNA adduct formation by its metabolites.

\section{CP-506 is selectively cytotoxic in several hypoxic 3D cell cultures}

As the hypoxia-selectivity of CP-506 is reliant on robust tissue penetration properties in vivo, we first compared the diffusion of CP-506 and PR-104A across HCT116 3D MCLs under hyperoxic conditions. When compared to PR-104A, CP-506 demonstrated greater stability in culture medium (Supplementary Fig. 4A) and a 2.2-fold higher diffusion coefficient $\left(2.75 \pm 0.43 \times 10^{-7}\right.$ vs. $1.26 \pm 0.14 \times 10^{-7} \mathrm{~cm}^{2} \mathrm{~s}^{1}$; Supplementary Fig. 4B and 4C). CP-506 thus exhibits excellent transport properties, a requirement for improved HAP performance. The superior aqueous stability of CP-506 also extended to human and mouse plasma stability, with no detectable loss of CP-506 (Supplementary Fig. 4D).

In line with data obtained in 2D cell cultures, we demonstrated that CP-506 had no cytotoxic effects in hyperoxic H460 MCLs. In anoxic MCLs, however, a concentration-dependent decrease in clonogenic cell survival was observed (Fig. 1F). In addition, H460 spheroids were exposed to CP-506, as spheroids naturally develop a diffusion-limited hypoxic core as they grow (Supplementary Fig. 4E). Growth inhibition was observed in hypoxic (day 11), but not normoxic (day 4) spheroids upon treatment with CP506 (Fig. 1G). Overall, these data confirm the hypoxia-selectivity of CP-506 using 3D in vitro cell culture models.

\section{Pharmacokinetic analysis of CP-506}


Modelling of plasma concentrations of CP-506 following IV and PO dosing ( $383 \mathrm{mg} / \mathrm{kg}$ ) (Supplementary Fig. $5 \mathrm{~A}$ ) demonstrated oral bioavailability $\left(F_{\text {abs }}\right)$ of $47 \%$ with a half-life $\left(T_{1 / 2}\right)$ of $0.92 \mathrm{~h}$ (Supplementary Table 4). Next, the plasma concentration-time profile of CP-506 was assessed using IP dosing (600 or 800 $\mathrm{mg} / \mathrm{kg} ; 50 \%$ and $67 \%$ of MTD, respectively), demonstrating maximum plasma concentrations $\left(\mathrm{C}_{\max }\right)$ of $246.2 \mu \mathrm{M}(600 \mathrm{mg} / \mathrm{kg})$ and $353.8 \mu \mathrm{M}(800 \mathrm{mg} / \mathrm{kg})$ with a calculated $\mathrm{T}_{1 / 2}$ of $0.54-0.58 \mathrm{~h}$. The area under the plasma concentration-time extrapolated to infinity $\left(\mathrm{AUC}_{0}-\right.$ inf $)$ was $206.7 \mu \mathrm{mol} . \mathrm{h} / \mathrm{L}(600 \mathrm{mg} / \mathrm{kg})$ and $276.3 \mu \mathrm{mol} . \mathrm{h} / \mathrm{L}$ (800 mg/kg) (Supplementary Fig. 5B).

\section{The antitumor effects of CP-506 are causally related to tumor oxygenation}

To investigate the capacity of HAP to preferentially sterilize hypoxic, radiation-resistant tumor cells, we first assessed the spatial relationship between tumor hypoxia and DNA damage in DMS114 xenograftbearing mice exposed to CP-506 (600 mg/kg, QD1, IP). CP-506 specifically induced DNA damage ( $\mathrm{HH} 2 \mathrm{AX}$ ) in hypoxic (pimonidazole-positive) regions (Fig. 2A and 2B). Secondly, we exposed H460 xenograftbearing mice to single-dose (10 Gy) irradiation (IR) followed by administration of CP-506 (900 or 1100 $\mathrm{mg} / \mathrm{kg}, \mathrm{QD} 1, \mathrm{IP})$. IR provided $0.95 \mathrm{LCK}$, which was amplified to 2.36 and $3.95 \mathrm{LCK}(\mathrm{p}<0.01)$, respectively, by subsequent administration of CP-506 (Fig. 2C). Administration of CP-506 alone resulted in 0.84 and 1.30 LCK, respectively. CP-506 thus demonstrated greater cell killing within the hypoxic, radiation-resistant tumor subpopulation than across the whole tumor cell population. Taken together, these data indicate that CP-506 preferentially exerts its antitumor effects in the hypoxic regions of the tumor.

The causal relationship between tumor oxygenation and antitumor effects of CP-506 was assessed in MDA-468 xenograft-bearing mice exposed to $7 \% \mathrm{O}_{2}, 21 \% \mathrm{O}_{2}$, or CarbN breathing conditions during which CP-506 (600 mg/kg, QD1, IP) was administered. Besides tumor growth monitoring, changes in hypoxic fraction (HF) were assessed in parallel animals injected with the hypoxia markers EF5 $1 \mathrm{~h}$ before the start of modified breathing conditions $\left(\mathrm{HF}_{\mathrm{EF} 5}\right)$ and pimonidazole $1 \mathrm{~h}$ before euthanasia, immediately after treatment $\left(\mathrm{HF}_{\text {pimo }}\right)$ (Fig. 2D). In vehicle-treated mice, no difference was observed between $\mathrm{HF}_{\text {pimo }}$ and $\mathrm{HF}_{\mathrm{EF} 5}$ (median $\mathrm{HF}_{\text {pimo }} / \mathrm{HF}_{\mathrm{EF} 5} 1.0$, interquartile range (IQR) 0.07 ) in animals exposed to $21 \% \mathrm{O}_{2}$ breathing conditions. Exposure to $7 \% \mathrm{O}_{2}$ increased $\mathrm{HF}_{\text {pimo }} / \mathrm{HF}_{\mathrm{EF} 5}$ (median 1.25, IQR 0.46) albeit not significantly ( $\mathrm{p}$ $=0.16$ ), whilst CarbN exposure significantly decreased $\mathrm{HF}_{\text {pimo }} / \mathrm{HF}_{\mathrm{EF} 5}$ (median 0.06, IQR 0.2; $\mathrm{p}<0.0001$ ) (Fig. 2E, Supplementary Fig. 6A). $\mathrm{HF}_{\text {pimo }} / \mathrm{HF}_{\mathrm{EF} 5}$ ratios of $\mathrm{CP}-506$-treated mice are presented in Supplementary Fig. 6B. Administration of CP-506 increased T4xSV to a higher extent in animals exposed to $7 \%(100.1 \pm 19.1$ vs. $51.4 \pm 14.8$ days, $p<0.0001)$ compared to animals exposed to $21 \% \mathrm{O}_{2}(87.5 \pm 15.7$ vs. $54.0 \pm 15.0$ days, $p<0.01)$ and CarbN (76.3 \pm 9.0 vs. $53.8 \pm 19.1$ days, $p=0.0781)$ (Fig. 2F). These data demonstrate a causal relationship between the antitumor effects of $\mathrm{CP}-506$ and tumor oxygenation status.

\section{CP-506 eradicates hypoxic tumor cells in a dose-dependent manner}


To further investigate the hypoxia-selective antitumor effects of CP-506, we first characterized the effects of CP-506 (800 mg/ $\mathrm{kg}$, QD5, IP) on the HF of MDA-231 xenografts. CP-506 decreased HF from $7.1 \pm 3.5 \%$ to $2.6 \pm 1.5 \%(p<0.05) 3$ days post-treatment, and from $7.3 \pm 2.6 \%$ to $3.8 \pm 3.1 \%(p=0.21) 10$ days posttreatment, indicating a time-dependent CP-506-mediated decrease in HF (Fig. 3A). Next, changes in HF in MDA-468 tumor xenografts were evaluated using a double hypoxia marker approach to investigate whether pimonidazole-positive cells were already present (pimonidazole- and CCl-103F-positive) at start of treatment, or if they were formed post treatment (pimonidazole positive and $\mathrm{CCl}$-103-negative). Different dosing regimens were tested (800 mg/kg, QD1; $400 \mathrm{mg} / \mathrm{kg}, \mathrm{QD} 3 ; 400 \mathrm{mg} / \mathrm{kg}, \mathrm{QD} 3 ;$ all IP). CP506 significantly decreased $\mathrm{HF}_{\mathrm{CCl}-103 \mathrm{~F}}$ and $\mathrm{HF}_{\text {pimo }}$ (Fig. $3 \mathrm{~B}$ and $3 \mathrm{C}$ ), and the ratio of $\mathrm{HF}_{\text {pimo }}$ to $\mathrm{HF}_{\mathrm{CCl}-103 \mathrm{~F}}$ (Fig. 3D), all in a dose-dependent manner, suggesting effective eradication of hypoxic tumor cells. Representative images are shown in Fig. 3E and 3F. Taken together, these data indicate that CP-506 eradicates hypoxic tumor cells.

\section{CP-506 exhibits dose-dependent and broad single-agent antitumor activity}

To characterize the single-agent activity of CP-506 and determine the optimal dose regimen, we first treated H69 small-cell lung cancer xenografts with various schedules of CP-506 (600 mg/kg, IP). An increased number of consecutive administrations was associated with increased depth of response. Repeated consecutive dose-schedules provided extended tumor control and were more effective than repeated intermittent dose-schedules (Supplementary Fig. 7A and 7B, Supplementary Table 5). Importantly, daily dosing was well-tolerated with transient body weight loss (Supplementary Fig. 7C). A dose regimen of a single CP-506 dose for five consecutive days (QD5) was selected for subsequent experiments. Next, we investigated whether CP-506 exhibited dose-dependent antitumor effects. MDA-468 xenograft-bearing mice were treated with increasing doses of CP-506 (200-800 mg/kg, QD5, IP). CP-506 exhibited dose-dependent TGD (T2xSV; Fig. 4A) and TGI at day 47 (final common monitoring time of all treatment arms) of $12.3 \%(200 \mathrm{mg} / \mathrm{kg}), 58.5 \%(400 \mathrm{mg} / \mathrm{kg}), 88.9 \%(600 \mathrm{mg} / \mathrm{kg})$, and $99.7 \%(800 \mathrm{mg} / \mathrm{kg})$. Vehicle-treated animals and animals treated with 600 or $800 \mathrm{mg} / \mathrm{kg}$ were monitored for a prolonged time period. Administration of $600 \mathrm{mg} / \mathrm{kg}$ CP-506 increased T4xSV significantly (163.2 \pm 33.3 days) compared to vehicle-treated tumors $(94.0 \pm 11.4$ days; $p<0.0001)$. Administration of $800 \mathrm{mg} / \mathrm{kg}$ resulted in complete and maintained long-term responses in 9 out of 10 animals (Fig. 4A and Supplementary Fig. 7D).

Finally, to broadly characterize the single-agent antitumor activity of CP-506 in vivo, animals bearing a wide range of human tumor xenografts were treated with CP-506 (600 or $800 \mathrm{mg} / \mathrm{kg}, \mathrm{QD} 5, \mathrm{IP})$. The tumor models were selected based on their in vitro sensitivity to CP-506, and were characterized by varying degrees of tumor hypoxia (Supplementary Fig. 8). In all models, CP-506 was well-tolerated with transient body weight loss during the treatment period. CP-506 effectively inhibited tumor growth in 13 out of 15 models tested, with TGIs ranging from 30.9-99.3\% (Table 2 and Fig. 4B), and significantly increased

$\mathrm{T} 4 \mathrm{xSV}$ in 12 out of 15 models tested, with calculated ERs ( $\mathrm{T} 4 \mathrm{xSV}_{\mathrm{CP}-506} / \mathrm{T}_{4 \mathrm{xSV}}$ vehicle $)$ ranging from 1.3 to 3.3 (Table 2 and Fig. 4C). In line with previous data, administration of CP-506 resulted in complete regression of 9 out of 10 animals bearing MDA-468 xenografts. T4xSV of BT-474 xenografts could not be 
determined due to short follow-up. Consistently, the only tumor models in which a complete lack of response was observed, MIA PaCa-2 and SW1990, demonstrated no detectable pimonidazole-positive hypoxia or expression of the endogenous hypoxia marker CAIX at the start of treatment (Supplementary Fig. 9), supporting the interpretation that the presence of hypoxia is an absolute requirement for CP-506 to exert its antitumor effects in vivo. 
Table 2

CP-506 exerts broad antitumor activity. CP-506 (600 (囚) or $\left.800\left({ }^{*}\right) \mathrm{mg} / \mathrm{kg}, \mathrm{IP}\right)$ were administered once per day for 5 consecutive days (QD5).

\begin{tabular}{|c|c|c|c|c|c|c|c|c|}
\hline Cell line & Cancer type & TGI\% & $\begin{array}{l}\text { M.S. } \\
\text { vehicle }\end{array}$ & $\begin{array}{l}\text { M.S. } \\
\text { CP- } \\
506\end{array}$ & $\begin{array}{l}\text { T4xSV - } \\
\text { vehicle }\end{array}$ & $\begin{array}{l}\text { T4XSV - } \\
\text { CP-506 }\end{array}$ & ER & $\begin{array}{l}\mathrm{p}- \\
\text { value }\end{array}$ \\
\hline $\begin{array}{l}\text { MIA } \\
\text { PaCa-2】 }\end{array}$ & $\begin{array}{l}\text { Pancreatic ductal } \\
\text { adenocarcinoma }\end{array}$ & -6.81 & 17.81 & 17.40 & 17.49 & 17.61 & 1.01 & 0.96 \\
\hline SW1990区 & $\begin{array}{l}\text { Pancreatic } \\
\text { adenocarcinoma }\end{array}$ & 0.51 & 15.50 & 16.31 & 16.19 & 16.38 & 1.01 & 0.88 \\
\hline $\begin{array}{l}\text { MDA- } \\
231 \rrbracket\end{array}$ & $\begin{array}{l}\text { Breast } \\
\text { adenocarcinoma }\end{array}$ & 30.88 & 16.42 & 20.72 & 15.925 & 21.32 & 1.34 & 0.0023 \\
\hline H1650区 & $\begin{array}{l}\text { Lung } \\
\text { adenocarcinoma }\end{array}$ & 30.97 & 8.36 & 12.06 & 8.08 & 11.94 & 1.48 & $<0.0001$ \\
\hline PANC-1》 & $\begin{array}{l}\text { Pancreatic ductal } \\
\text { adenocarcinoma }\end{array}$ & 36.91 & 14.80 & 20.61 & 15.58 & 21.53 & 1.38 & 0.0003 \\
\hline BT-474ه & $\begin{array}{l}\text { Breast ductal } \\
\text { adenocarcinoma }\end{array}$ & 37.29 & 18.58 & N/A & 17.68 & N/A & $\mathrm{N} / \mathrm{A}$ & $\mathrm{N} / \mathrm{A}$ \\
\hline $\begin{array}{l}\text { DMS- } \\
114 \rrbracket\end{array}$ & $\begin{array}{l}\text { Small cell lung } \\
\text { carcinoma }\end{array}$ & 44.30 & 16.16 & 27.40 & 15.81 & 27.48 & 1.74 & $<.0001$ \\
\hline H69ם & $\begin{array}{l}\text { Small cell lung } \\
\text { carcinoma }\end{array}$ & 74.55 & 10.34 & 21.44 & 9.72 & 20.92 & 2.15 & $\begin{array}{l}< \\
0.0001\end{array}$ \\
\hline $\begin{array}{l}\text { MDA- } \\
436 \rrbracket\end{array}$ & $\begin{array}{l}\text { Breast } \\
\text { adenocarcinoma }\end{array}$ & 88.29 & 17.08 & 53.99 & 16.95 & 51.83 & 3.06 & $\dot{0} 0001$ \\
\hline SiHa* & $\begin{array}{l}\text { Cervix squamous } \\
\text { cell carcinoma }\end{array}$ & 39.98 & 13.19 & 19.73 & 13.50 & 19.88 & 1.47 & 0.022 \\
\hline HCT116* & $\begin{array}{l}\text { Colorectal } \\
\text { carcinoma }\end{array}$ & 41.66 & 16.77 & 34.23 & 20.36 & 33.54 & 1.65 & 0.0095 \\
\hline $\mathrm{H} 460$ * & $\begin{array}{l}\text { Large cell lung } \\
\text { carcinoma }\end{array}$ & 44.75 & 4.31 & 8.76 & 4.58 & 10.52 & 2.30 & 0.0026 \\
\hline$A 2780 *$ & $\begin{array}{l}\text { Ovarian } \\
\text { adenocarcinoma }\end{array}$ & 70.08 & 5.12 & 17.78 & 5.55 & 18.52 & 3.34 & 0.0014 \\
\hline $\begin{array}{l}\text { MDA- } \\
231^{\star}\end{array}$ & $\begin{array}{l}\text { Breast } \\
\text { adenocarcinoma }\end{array}$ & 83.59 & 23.17 & 56.92 & 25.27 & 59.39 & 2.35 & 0.0004 \\
\hline $\begin{array}{l}\text { MDA- } \\
468^{\star}\end{array}$ & $\begin{array}{l}\text { Breast } \\
\text { adenocarcinoma }\end{array}$ & 99.32 & 73.12 & N/A & 73.09 & N/A & $\mathrm{N} / \mathrm{A}$ & $\mathrm{N} / \mathrm{A}$ \\
\hline
\end{tabular}




\section{Baseline tumor hypoxia and in vitro sensitivity are correlated with the treatment response to CP-506}

To identify which factors contribute to the tumoral response to CP-506, defined as ER or SGD, a multivariate linear regression analysis was performed. ER was positively correlated with baseline HF ( $p<$ $0.0005)$ and negatively correlated with the in vitro sensitivity (anoxic $\left.I C_{50}\right)(p<0.0005)$. A higher $C P-506$ dose was associated with a higher ER $(p<0.0001)$. Similar results were obtained using SGD as outcome variable. Using these significantly variables, a nomogram was constructed allowing ER prediction (Supplementary Fig. 10). Taken together, these data demonstrate that the degree of tumor hypoxia and the in vitro sensitivity to $\mathrm{CP}-506$ are significantly correlated to the treatment response.

\section{Discussion}

In the present study, we evaluated the therapeutic potential of the hypoxia-activated DNA crosslinking prodrug CP-506, designed to lack off-mechanism aerobic metabolism unlike its predecessor PR-104 (23), whilst concurrently possessing superior physicochemical and pharmacokinetic properties, including improved solubility, stability, tissue diffusion and oral bioavailability.

CP-506 exhibited a one-electron affinity $\left(E^{0}\right)$ optimal for reduction by human endogenous single-electron oxidoreductases, and a back-oxidation rate constant that indicates efficient competition with molecular oxygen. Further, direct measurements of metabolism and cytotoxicity of CP-506 using stirred cell suspensions with accurate, real-time measurements of soluble $\mathrm{O}_{2}$ concentrations confirmed the strict hypoxia-dependent bioactivation of CP-506, which was completely inhibited at $\mathrm{O}_{2}$ concentrations above 1 $\mu \mathrm{M}\left(0.1 \% \mathrm{O}_{2}\right)$. CP-506 demonstrates an unusually steep k-curve providing optimal selectivity for pathological hypoxia. Consequently, whilst its $\mathrm{KO}_{2}$ value is only 2 -fold lower than that of tirapazamine (38), CP-506 is fully and completely inhibited at $\mathrm{O}_{2}$ concentrations 100 -fold lower $(1 \mu \mathrm{M}$ vs. $100 \mu \mathrm{M} \mathrm{O}$, respectively) (Supplementary Fig. 11).

In a wide variety of in vitro 2D monolayer cell cultures of different origin, we showed CP-506 exerts its cytotoxic effects selectively under anoxic conditions, with HCRs ranging from 4 to 203 . These results are in line with HCRs previously reported and confirmed here in side-by-side analysis for PR104A, ranging from 6 to 160 (18), but lower when compared the HAP evofosfamide, with reported HCRs up to 600 (39). This finding may be explained by differences in the bystander effects of CP-506 and evofosfamide: the bystander effect of CP-506 allows escape and dilution of its metabolites into surrounding medium, whilst the activated metabolite of evofosfamide remains largely entrapped in the cell of origin (17), exerting maximal cytotoxicity at the source of metabolism and giving rise to amplified HCRs. The discordant influence of the bystander effect on these 2D assays therefore limits accurate comparison of the hypoxiaselectivity of HAPs with diverse physicochemical properties. To address this experimental limitation, we confirmed the hypoxia-selectivity of CP-506 in several 3D in vitro cell culture models, which mimic the structure of the in vivo tumor environment more closely than 2D monolayers (40). 
In vivo, CP-506 demonstrated good plasma exposures at well-tolerated doses leading to potent singleagent antitumor activity in 13 hypoxic xenograft models tested. In line with this, HAPs such as PR-104 (18) and evofosfamide (7) have also displayed strong monotherapeutic antitumor effects. However, it is now understood that the antitumor effects of PR-104 and evofosfamide are not solely due to their HAP behavior: PR-104 single-agent activity is dominated by the unforeseen oxygen-independent metabolism by AKR1C3 (23), whilst the single-agent activity of evofosfamide is mediated by background activation in normoxic tumor regions likely due to inadequate inhibition by $\mathrm{O}_{2}(17)$. Importantly, we establish here that $\mathrm{CP}-506$ is resistant to AKR1C3 reductive metabolism and is fully quenched by $1 \mu \mathrm{M} \mathrm{O} \mathrm{O}_{2}$. PR-104 is not a substrate for the murine orthologues, resulting in favorable murine toxicokinetics (26) that failed to translate to human dose tolerance in Phase I trials $(21,22)$. Thus, without the confounding factor of human aerobic AKR1C3 metabolism, it is reasonable to anticipate that the interspecies allometric scaling of CP-506 will extrapolate in a predictable manner (41).

Several factors have been proposed to influence the in vivo antitumor effects of HAPs (42), including the extent of tumor hypoxia. Indeed, in a monotherapy study employing 8 different xenograft models, Sun et al. found a good correlation between baseline tumor hypoxia and evofosfamide (7), confirmed later in a different study employing modified breathing strategies (9). Here, by using similar modified breathing strategies, we demonstrated that the efficacy of CP-506 increased as function of HF in MDA-468 tumor xenografts. Moreover, in a multivariate linear regression analysis employing data of 15 different tumor models, we showed that pretreatment tumor hypoxia as well as intrinsic cellular sensitivity to CP-506 strongly correlated with tumor response. Consistently, our results also indicate that hypoxia is an absolute prerequisite for CP-506 to exert its antitumor effects as the MIA PaCa-2 and SW1990 pancreatic tumor models, despite their significant cellular sensitivity to $\mathrm{CP}-506$ in vitro (Table 1), exhibited a complete lack of response in vivo associated with total absence of hypoxia. Further supportive of the hypoxia-mediated mechanism of action of CP-506 is the spatial relationship of tumor hypoxia and accumulated DNA damage, a greater cell kill observed within the radiation resistant, hypoxic tumor subpopulation, and a dose-dependent decrease in tumor HF.

Since HAPs require an initial activation step by endogenous one-electron oxidoreductases, the levels and catalytic activity of prodrug-activating nitroreductases are a second factor likely contributing to HAP efficacy $(35,43,44)$. Here, we identified cytochrome P450 oxidoreductase (POR), methionine synthase reductase (MTRR), novel diflavin oxidoreductase 1 (NDOR1) and inducible nitric oxide synthase (NOS2A) as likely candidates, although it should be noted that one-electron oxidoreductases responsible for HAP activation can exhibit functional redundancy (42). Additionally, the intrinsic sensitivity to the activated, cytotoxic metabolites of CP-506 is likely to influence its antitumor effects. For PR-104 and evofosfamide, it has been shown that tumors deficient in homologous recombination (HR) are markedly more sensitive to HAP treatment $(20,39,45)$. As CP-506 exerts its cytotoxicity by the formation of DNA interstrand crosslinks and subsequent induction of DNA double-strand breaks following replication fork stalling, the integrity of DNA repair mechanisms such as the above-mentioned HR and the Fanconi anemia pathway is likely an important factor in determining the efficacy of CP-506 (46). Experiments to investigate the 
influence of oxidoreductase expression and activity, and integrity of the DNA repair response on the sensitivity to CP-506 are currently ongoing.

To date, HAP clinical trials have yielded disappointing results, with a lack of patient stratification accountable for their failure (3). Therefore, gaining a greater mechanistic understanding of the factors and their complex interplay in determining the antitumor effects of CP-506 is essential for successful clinical translation. Despite the clear monotherapeutic activity of CP-506, it should be noted that the therapeutic efficacy of HAPs is predicted to be the greatest when combined with other treatment modalities such as chemo- and radiotherapy (47). Since HAPs mainly target the hypoxic tumor cells, the therapeutic potential can theoretically be enhanced by a different treatment modality that kills aerobic cells. Currently, experiments assessing the effects of the combination of CP-506, radiotherapy and/or immunotherapy are ongoing.

\section{Conclusion}

CP-506 is a novel hypoxia-activated prodrug with electron-affinic properties specifically designed to confer a strict k-curve that is appropriately offset by a local bystander effect (36). Drug-like properties include aqueous solubility, oral bioavailability, plasma stability and absence of off-mechanism activation by the human aerobic reductase AKR1C3. We confirm that CP-506 selectively kills hypoxic tumor cells in vitro, and this activity is related to selective formation of DNA adducts under hypoxic conditions. CP-506 also inhibits growth and clonogenic survival specifically in hypoxic spheroids. In vivo, CP-506 demonstrates antitumor effects in a broad range of tumor xenograft models. A causal relationship is established between tumor oxygenation and antitumor effects of CP-506 in MDA-468 xenografts as well as significant correlation between baseline hypoxia and tumor response across different tumor types using a multivariate approach. Several additional factors besides tumor hypoxia are proposed to influence the tumor response to CP-506. Generating a strong mechanistic understanding of CP-506 will facilitate the development of predictive or prognostic biomarkers of response critical for successful clinical translation. It is anticipated that the upcoming clinical evaluation of CP-506 will utilize these newly discovered biomarkers of response to guide future patient selection.

\section{Abbreviations}

AKR1C3, aldo-keto reductases 1C3; CCl-103F, 1-(1,1,1,3,3,3-hexafluoropropan-2-yloxy)-3-(2-nitroimidazol-1yl)propan-2-ol; DMSO, dimethyl sulfoxide; $\left(E^{O}\right)$, one-electron affinity; EF5, 2-(2-Nitro-1H-imidazol-1-yl)-N(2,2,3,3,3-pentafluoropropyl)acetamide; HAP, Hypoxia-activated prodrug; HCR, hypoxia cytotoxicity ratio; IV, intravenous; IP, intraperitoneal; LC-MS/MS, liquid-chromatography with tandem mass spectrometry; MTRR, methionine synthase reductase; NDOR1, novel diflavin oxidoreductase 1; NOS2A, inducible nitric oxide synthase; NOS3, endothelial nitric oxide synthase; PCR, polymerase chain reaction; POR, cytochrome P450 oxidoreductase; PE, plating efficiency; SRB, Sulforhodamine B; WFI, water for injection.

\section{Declarations}


Ethics approval and consent to participate: All animal studies were approved by the corresponding Institutional Animal Ethics Committees.

Consent for publication: All authors have reviewed in full and approved the manuscript for publication

Availability of data and material: All data and non-proprietary materials are available on request

Competing interests: AH, ST, and SD have been prior employees of Convert Pharmaceuticals. PL reports within and outside the submitted work - grants or sponsored research agreements from Varian Medical, Oncoradiomics, ptTheragnostic/DNAmito, and Health Innovation Ventures. He received an advisor/presenter fee and/or reimbursements of travel costs/external grant writing fee and/or kind manpower contribution from Oncoradiomics, BHV, Merck, Varian, Elekta, ptTheragnostic, and Convert Pharmaceuticals. PL has shares in the company Oncoradiomics SA, Convert Pharmamaceuticals, and The Medical Cloud Company SPRL, and is co-inventor of two issues patents with royalties on radiomics (PCT/NL2014/050248, PCT/NL2014/050728) licensed to Oncoradiomics, one issue patent on mtDNA (PCT/EP2014/059089) licensed to ptTheragnostic/DNAmito, three non-patented inventions (software) licensed to ptTheragnostic/DNAmito and Oncoradiomics and Health Innovation Ventures, and three nonissues, non-licensed patents on Deep Learning-Radiomics and LSRT (N2024482, N2024889, N2024889). $\mathrm{AH}, \mathrm{JT}$, and LD have shares in the company Convert Pharmaceuticals, and LD has a non-issue, nonlicensed patent on LSRT (N2024889). JS and AP have previously served as scientific consultants to Convert Pharmaceuticals. JBS, AVP, AMM, AA and CPG are co-inventors on patents assigned to Health Innovation Ventures (PCT/WO 2014031012 A1).

Funding: This work was financially supported by a Health Research Council of New Zealand (project grant 17/255) and by the ERC advanced grant HYPOXIMMUMO (ERC-ADG-2015, $n^{\circ} 694812$ ). The research conducted in this paper has been funded by the Walloon Region of Belgium through the SPW DG Economie, Emploi, Recherche, its implementing body for regional research and innovation. The authors also acknowledge financial support from the EUROSTARS grant COMPACT (12053) and the Cancer Society Auckland Northland (CSAN).

Authors' contributions: AW, VJP, and RN contributed equally to this work, including designing and performing biological experiments, prepared the figures, and drafting the manuscript. $A Y, A M M, X L, Z F, A A$, RFA, KOH, CPG, SD, ST, AH, SB, JBS, JT, LD, AVP, and PL analyzed biological experiments, prepared the figures, edited and revised the manuscript. SK, AA, RFA, NS, JBS, and AVP performed chemistry experiments; AY, EL, DM, AMM, NL, RB, XL, ZF, RFA, KOH, MRB, MRA, CPG, SD, SB, JBS, LD, and AVP performed biological experiments. Data analysis was performed by $A Y, E L, D M, A M M, N L, R B, X L, S K, A J$, AA, RFA, KOH, MRB, MRA, CPG, SD, ST, MS, SB, JT, LD, and AVP. AY, EL, AJ, AA, RFA, KOH, MRB, SB, JBS, JT, LD, AVP, and PL helped write the manuscript; AY, EL, DM, AMM, AJ, AA, RFA, KOF, MRB, MRA, SD, ST, $A H, S B, J B S, L T, L D, A V P$, and PL revised the manuscript. $L D, A V P$, and PL were responsible for the scientific content and technical accuracy of the manuscript including final approval of the revised the manuscript. 
Acknowledgements: Prof. M. Losen and Ms. H. Steinbusch from the Department of Psychiatry and Neuropsychology, School for Mental Health and Neuroscience, Maastricht University are greatly acknowledged for their technical support with microscope image acquisition.

\section{References}

1. Hockel M, Vaupel P. Tumor hypoxia: definitions and current clinical, biologic, and molecular aspects. J Natl Cancer Inst. 2001/02/22. 2001;93(4):266-76.

2. Vaupel $P$, Mayer A. Hypoxia in cancer: significance and impact on clinical outcome. Cancer Metastasis Rev. 2007/04/19. 2007;26(2):225-39.

3. Spiegelberg L, Houben R, Niemans R, de Ruysscher D, Yaromina A, Theys J, et al. Hypoxia-activated prodrugs and (lack of) clinical progress: The need for hypoxia-based biomarker patient selection in phase III clinical trials. Clin Transl Radiat Oncol. 2019/02/09. 2019;15:62-9.

4. Wilson WR, Hay MP. Targeting hypoxia in cancer therapy. Nat Rev Cancer. 2011/05/25. 2011;11(6):393-410.

5. Phillips RM. Targeting the hypoxic fraction of tumours using hypoxia-activated prodrugs. Cancer Chemother Pharmacol. 2016/01/27. 2016;77(3):441-57.

6. Guise CP, Mowday AM, Ashoorzadeh A, Yuan R, Lin W-H, Wu D-H, et al. Bioreductive prodrugs as cancer therapeutics: targeting tumor hypoxia. Chin J Cancer. 2014 Feb;33(2):80-6.

7. Sun JD, Liu Q, Wang J, Ahluwalia D, Ferraro D, Wang Y, et al. Selective tumor hypoxia targeting by hypoxia-activated prodrug TH-302 inhibits tumor growth in preclinical models of cancer. Clin Cancer Res. 2011/12/21. 2012;18(3):758-70.

8. Sun JD, Ahluwalia D, Liu Q, Li W, Wang Y, Meng F, et al. Combination treatment with hypoxiaactivated prodrug evofosfamide (TH-302) and mTOR inhibitors results in enhanced antitumor efficacy in preclinical renal cell carcinoma models. Am J Cancer Res. 2015/09/04. 2015;5(7):213955.

9. Peeters SG, Zegers CM, Biemans R, Lieuwes NG, van Stiphout RG, Yaromina A, et al. TH-302 in Combination with Radiotherapy Enhances the Therapeutic Outcome and Is Associated with Pretreatment [18F]HX4 Hypoxia PET Imaging. Clin Cancer Res. 2015/03/26. 2015;21(13):2984-92.

10. Yaromina A, Granzier M, Biemans R, Lieuwes N, van Elmpt W, Shakirin G, et al. A novel concept for tumour targeting with radiation: Inverse dose-painting or targeting the "Low Drug Uptake Volume." Radiother Oncol. 2017/05/16. 2017;124(3):513-20.

11. Ganjoo KN, Cranmer LD, Butrynski JE, Rushing D, Adkins D, Okuno SH, et al. A phase I study of the safety and pharmacokinetics of the hypoxia-activated prodrug TH-302 in combination with doxorubicin in patients with advanced soft tissue sarcoma. Oncology. 2011/06/01. 2011;80(12):50-6.

12. Borad MJ, Reddy SG, Bahary N, Uronis HE, Sigal D, Cohn AL, et al. Randomized Phase II Trial of Gemcitabine Plus TH-302 Versus Gemcitabine in Patients With Advanced Pancreatic Cancer. J Clin 
Oncol. 2014/12/17. 2015;33(13):1475-81.

13. Cutsem E Van, Lenz H-J, Furuse J, Tabernero J, Heinemann V, loka T, et al. MAESTRO: A randomized, double-blind phase III study of evofosfamide (Evo) in combination with gemcitabine (Gem) in previously untreated patients (pts) with metastatic or locally advanced unresectable pancreatic ductal adenocarcinoma (PDAC). J Clin Oncol. 2016;34(15_suppl):4007.

14. Tap WD, Papai Z, Van Tine BA, Attia S, Ganjoo KN, Jones RL, et al. Doxorubicin plus evofosfamide versus doxorubicin alone in locally advanced, unresectable or metastatic soft-tissue sarcoma (TH CR-406/SARC021): an international, multicentre, open-label, randomised phase 3 trial. Lancet Oncol. 2017/06/28. 2017;18(8):1089-103.

15. Higgins JP, Sarapa N, Kim J, Poma E. Unexpected pharmacokinetics of evofosfamide observed in phase III MAESTRO study. J Clin Oncol. 2018;36(15_suppl):2568.

16. Millis KK, Colvin ME, Shulmanroskes EM, Ludeman SM, Colvin OM, Gamcsik MP. Comparison of the Protonation of Isophosphoramide Mustard and Phosphoramide Mustard. J Med Chem. 1995;38(12):2166-75.

17. Hong CR, Wilson WR, Hicks KO. An Intratumor Pharmacokinetic/Pharmacodynamic Model for the Hypoxia-Activated Prodrug Evofosfamide (TH-302): Monotherapy Activity is Not Dependent on a Bystander Effect. Neoplasia. 2018/12/29. 2019;21(2):159-71.

18. Patterson A V, Ferry DM, Edmunds SJ, Gu Y, Singleton RS, Patel K, et al. Mechanism of action and preclinical antitumor activity of the novel hypoxia-activated DNA cross-linking agent PR-104. Clin Cancer Res. 2007/07/04. 2007;13(13):3922-32.

19. Abbattista MR, Jamieson SM, Gu Y, Nickel JE, Pullen SM, Patterson A V, et al. Pre-clinical activity of PR-104 as monotherapy and in combination with sorafenib in hepatocellular carcinoma. Cancer Biol Ther. 2015/04/15. 2015;16(4):610-22.

20. Hunter FW, Hsu HL, Su J, Pullen SM, Wilson WR, Wang J. Dual targeting of hypoxia and homologous recombination repair dysfunction in triple-negative breast cancer. Mol Cancer Ther. 2014/09/07. 2014;13(11):2501-14.

21. Jameson MB, Rischin D, Pegram M, Gutheil J, Patterson A V, Denny WA, et al. A phase I trial of PR104 , a nitrogen mustard prodrug activated by both hypoxia and aldo-keto reductase $1 \mathrm{C} 3$, in patients with solid tumors. Cancer Chemother Pharmacol. 2009/12/17. 2010;65(4):791-801.

22. McKeage MJ, Gu Y, Wilson WR, Hill A, Amies K, Melink TJ, et al. A phase I trial of PR-104, a preprodrug of the bioreductive prodrug PR-104A, given weekly to solid tumour patients. BMC Cancer. 2011/10/11. 2011;11:432.

23. Guise CP, Abbattista MR, Singleton RS, Holford SD, Connolly J, Dachs GU, et al. The bioreductive prodrug PR-104A is activated under aerobic conditions by human aldo-keto reductase 1C3. Cancer Res. 2010/02/11. 2010;70(4):1573-84.

24. Birtwistle J, Hayden RE, Khanim FL, Green RM, Pearce C, Davies NJ, et al. The aldo-keto reductase AKR1C3 contributes to 7,12-dimethylbenz(a)anthracene-3,4-dihydrodiol mediated oxidative DNA 
damage in myeloid cells: implications for leukemogenesis. Mutat Res. 2009/01/24. 2009;662(12):67-74.

25. Konopleva M, Thall PF, Yi CA, Borthakur G, Coveler A, Bueso-Ramos C, et al. Phase I/II study of the hypoxia-activated prodrug PR104 in refractory/relapsed acute myeloid leukemia and acute lymphoblastic leukemia. Haematologica. 2015 Jul;100(7):927-34.

26. Patel K, Choy SS, Hicks KO, Melink TJ, Holford NH, Wilson WR. A combined pharmacokinetic model for the hypoxia-targeted prodrug PR-104A in humans, dogs, rats and mice predicts species differences in clearance and toxicity. Cancer Chemother Pharmacol. 2010/08/05. 2011;67(5):114555 .

27. Smaill JB, Patterson A V, Ashoorzadeh A, Guise CP, Mowday AM, Ackerley DF, et al. Novel Prodrugs and Methods of Use Thereof. PCT Int.Appl.; WO2014031012A1, 2014.

28. Smaill JB, Patterson A V, Ashoorzadeh A, Guise CP, Mowday AM, Ackerley DF, et al. Prodrugs and Methods of Use Thereof. U.S. Pat. Appl. Publ.; US 20180148463 A1, 2018.

29. Heyerick A, Deschoemaeker S, Thiolloy S, Tersago D, Lambin P. Prodrugs and Medical Uses Thereof. WO 2019229195 A1.

30. Gu Y, Atwell GJ, Wilson WR. Metabolism and excretion of the novel bioreductive prodrug PR-104 in mice, rats, dogs, and humans. Drug Metab Dispos. 2009/12/19. 2010;38(3):498-508.

31. Gu Y, Tingle MD, Wilson WR. Glucuronidation of anticancer prodrug PR-104A: species differences, identification of human UDP-glucuronosyltransferases, and implications for therapy. J Pharmacol Exp Ther. 2011/03/24. 2011;337(3):692-702.

32. Atwell GJ, Denny WA. Synthesis of $3 \mathrm{H}$ - and $2 \mathrm{H} 4$-labelled versions of the hypoxia-activated preprodrug 2-[(2-bromoethyl)-2,4-dinitro-6- [[[2-(phosphonooxy)ethyl]amino]carbonyl]anilino]ethyl methanesulfonate (PR-104). J Label Compd Radiopharm. 2007 Jan 1;50(1):7-12.

33. Yang S, Atwell G, Denny W. Synthesis of asymmetric halomesylate mustards with aziridineethanol/alkali metal halides: application to an improved synthesis of the hypoxia prodrug PR-104. Tetrahedron. 2007 Jun 18;63:5470-6.

34. Anderson RF, Yadav P, Patel D, Reynisson J, Tipparaju SR, Guise CP, et al. Characterisation of radicals formed by the triazine 1,4-dioxide hypoxia-activated prodrug, SN30000. Org Biomol Chem. 2014;12(21):3386-92.

35. Guise CP, Abbattista MR, Tipparaju SR, Lambie NK, Su J, Li D, et al. Diflavin oxidoreductases activate the bioreductive prodrug PR-104A under hypoxia. Mol Pharmacol. 2011/10/11. 2012;81(1):31-40.

36. Wilson WR, Hicks KO, Pullen SM, Ferry DM, Helsby NA, Patterson A V. Bystander effects of bioreductive drugs: potential for exploiting pathological tumor hypoxia with dinitrobenzamide mustards. Radiat Res. 2007/05/26. 2007;167(6):625-36.

37. Yahyanejad S, van Hoof SJ, Theys J, Barbeau LM, Granton P V, Paesmans K, et al. An image guided small animal radiation therapy platform (SmART) to monitor glioblastoma progression and therapy response. Radiother Oncol. 2015/07/15. 2015;116(3):467-72. 
38. Hicks KO, Myint H, Patterson A V, Pruijn FB, Siim BG, Patel K, et al. Oxygen dependence and extravascular transport of hypoxia-activated prodrugs: comparison of the dinitrobenzamide mustard PR-104A and tirapazamine. Int J Radiat Oncol Biol Phys. 2007/09/18. 2007;69(2):560-71.

39. Meng F, Evans JW, Bhupathi D, Banica M, Lan L, Lorente G, et al. Molecular and cellular pharmacology of the hypoxia-activated prodrug TH-302. Mol Cancer Ther. 2011/12/08. 2012;11(3):740-51.

40. Langhans SA. Three-Dimensional in Vitro Cell Culture Models in Drug Discovery and Drug Repositioning. Vol. 9, Frontiers in Pharmacology. 2018. p. 6.

41. Nair $A B$, Jacob S. A simple practice guide for dose conversion between animals and human. $J$ basic Clin Pharm. 2016 Mar;7(2):27-31.

42. Hunter FW, Wouters BG, Wilson WR. Hypoxia-activated prodrugs: paths forward in the era of personalised medicine. Br J Cancer. 2016/04/14. 2016;114(10):1071-7.

43. Guise CP, Wang AT, Theil A, Bridewell DJ, Wilson WR, Patterson A V. Identification of human reductases that activate the dinitrobenzamide mustard prodrug PR-104A: a role for NADPH:cytochrome P450 oxidoreductase under hypoxia. Biochem Pharmacol. 2007/07/25. 2007;74(6):810-20.

44. Hunter FW, Young RJ, Shalev Z, Vellanki RN, Wang J, Gu Y, et al. Identification of P450 Oxidoreductase as a Major Determinant of Sensitivity to Hypoxia-Activated Prodrugs. Cancer Res. 2015/08/25. 2015;75(19):4211-23.

45. Gu Y, Patterson A V, Atwell GJ, Chernikova SB, Brown JM, Thompson LH, et al. Roles of DNA repair and reductase activity in the cytotoxicity of the hypoxia-activated dinitrobenzamide mustard PR104A. Mol Cancer Ther. 2009/06/11. 2009;8(6):1714-23.

46. Deans AJ, West SC. DNA interstrand crosslink repair and cancer. Nat Rev Cancer. 2011/06/28. 2011;11(7):467-80.

47. Hamis S, Kohandel M, Dubois LJ, Yaromina A, Lambin P, Powathil GG. Combining hypoxia-activated prodrugs and radiotherapy in silico: Impact of treatment scheduling and the intra-tumoural oxygen landscape. PLoS Comput Biol. 2020 Aug;16(8):e1008041.

\section{Figures}



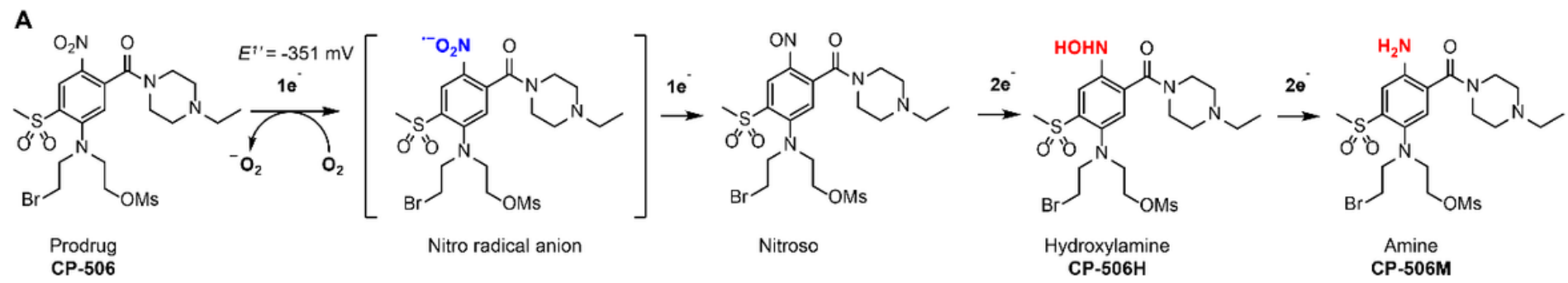

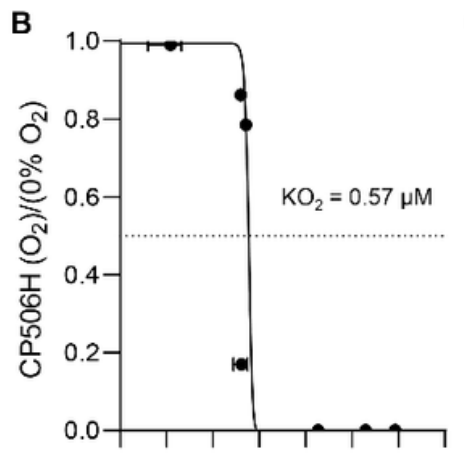

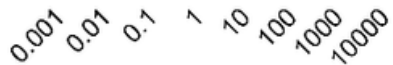

Soluble $\mathrm{O}_{2}$ concentration ( $\left.\mu \mathrm{M}\right)$

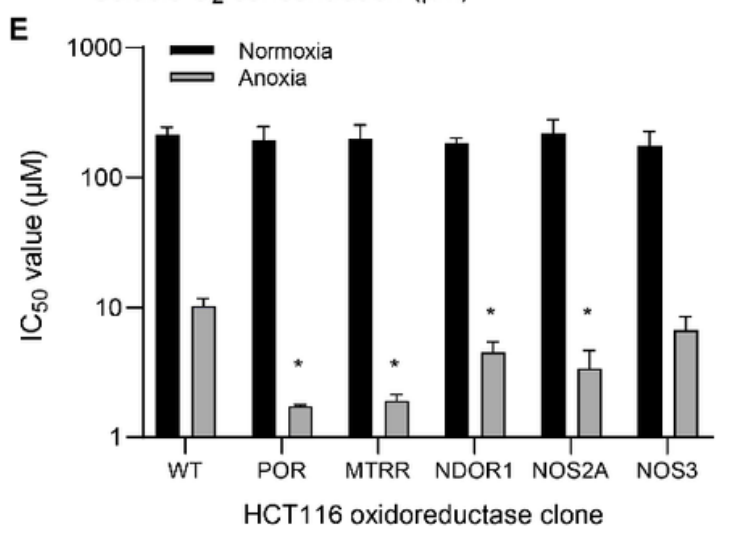

C

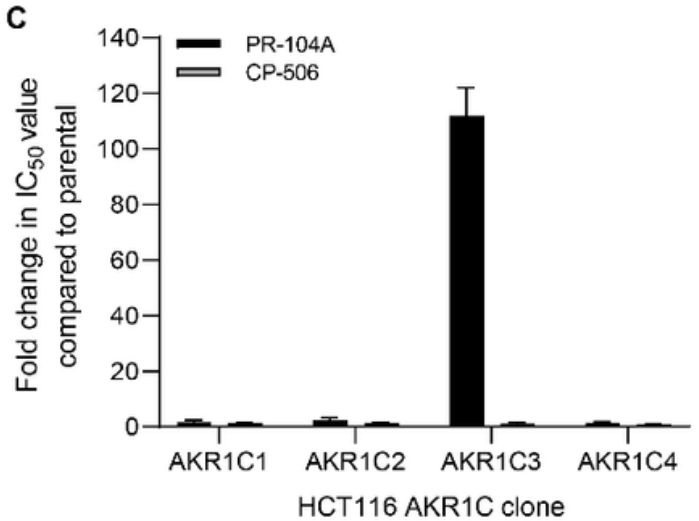

$\mathbf{F}$

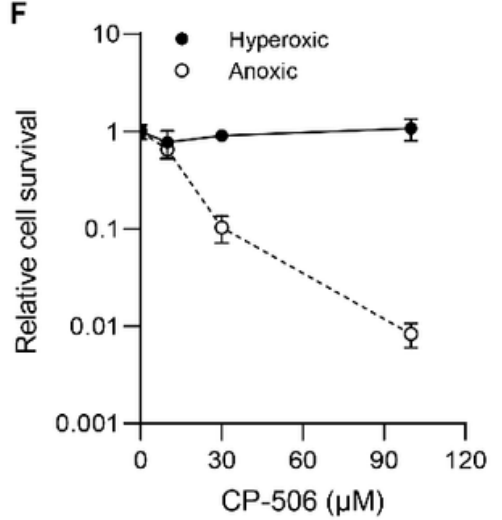

D
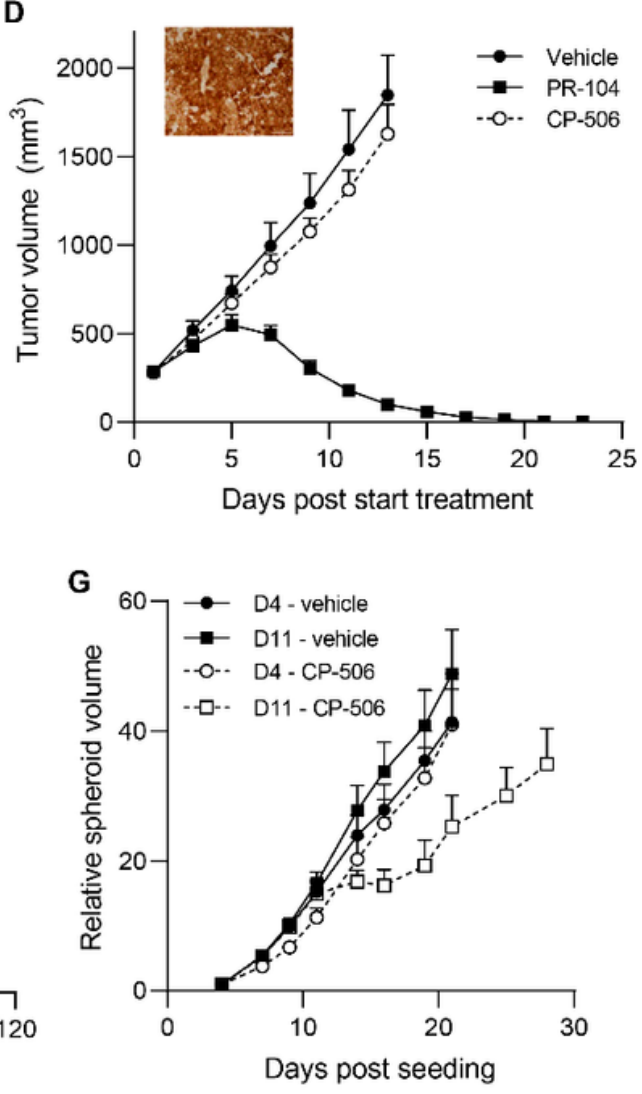

Figure 1

CP-506 is resistant to aerobic activation. (A) Proposed mechanism of activation of CP-506. (B) The 02dependence of CP-506 metabolism (CP-506H formation). (C) Fold-change in IC50 values of AKR1Cexpressing HCT116 cells exposed to PR-104A or CP-506 compared to parental cells. (D) Tumor growth of PR-104- and CP-506-treated SNU-398 tumors engineered to express human AKR1C3 as confirmed by immunohistochemical detection of AKR1C3 on FFPE tumor sections (image insert). (E) Comparative normoxic and anoxic IC50 values of HCT116 cell overexpressing human diflavin oxidoreductases; POR (5.9-fold; $p=0.008)$, MTRR (5.4-fold; $p=0.008)$, NDOR1 (2.3-fold; $p=0.016$ ) and NOS2A (3.0-fold; $p=$ $0.010)$, NOS3 $(p=0.16)$. ( $F)$ Clonogenic cell survival of H460 MCLs with differential oxygenation status after $4 \mathrm{~h}$ CP-506 exposure. (G) Effect of CP-506 on growth of H460 spheroids with differential hypoxic status. Spheroids were treated with CP-506 $(250 \mu \mathrm{M})$ at day 4 (D4; normoxic) or day 11 (D11; hypoxic), after which spheroid grow was monitored. Data are presented as mean \pm SEM. 
A

Pimonidazole

YH2AX

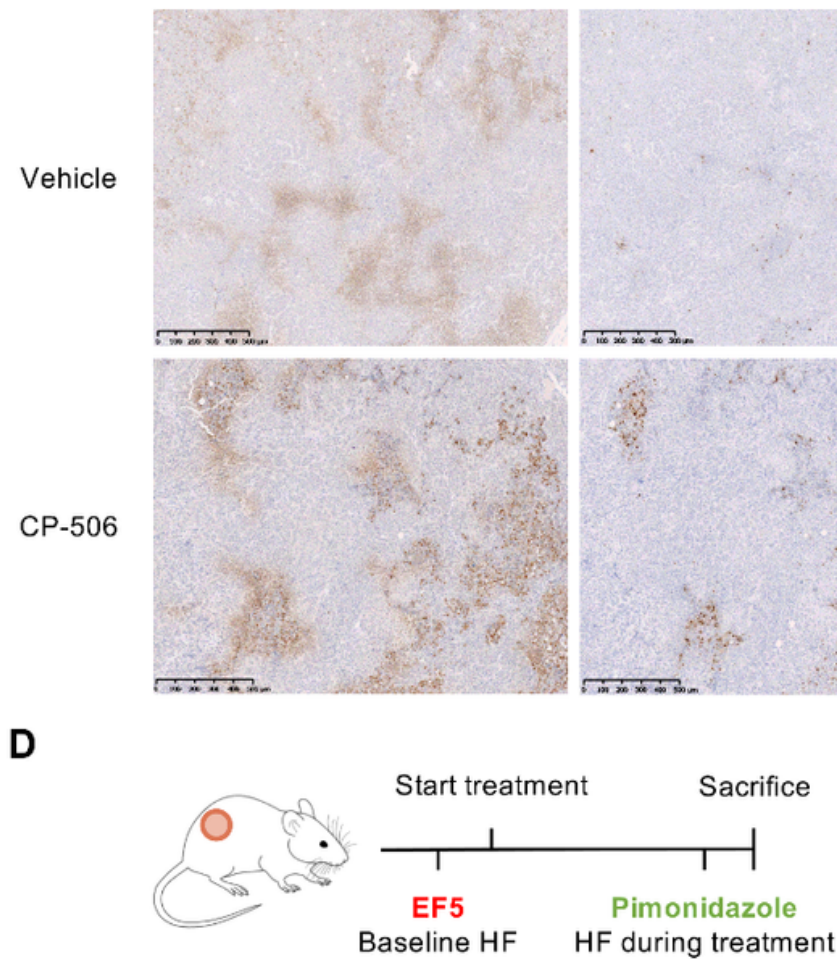

$\mathbf{E}$

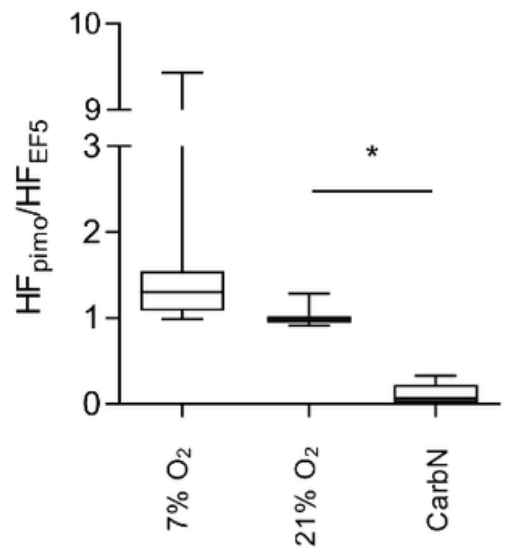

B

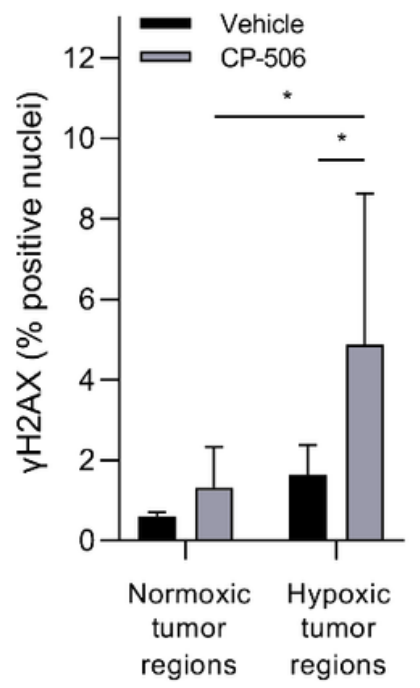

C

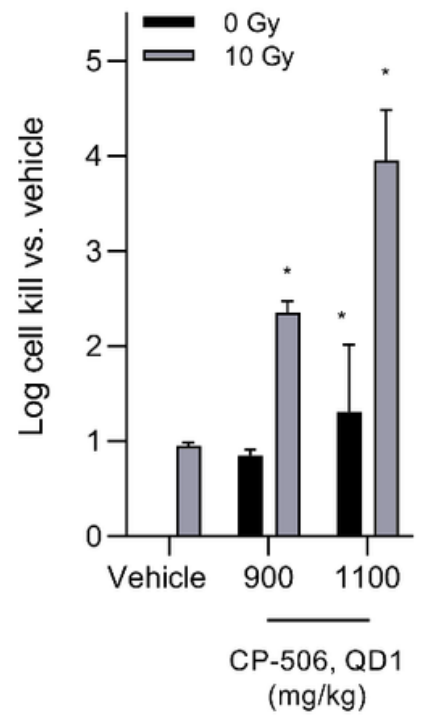

$\mathbf{F}$

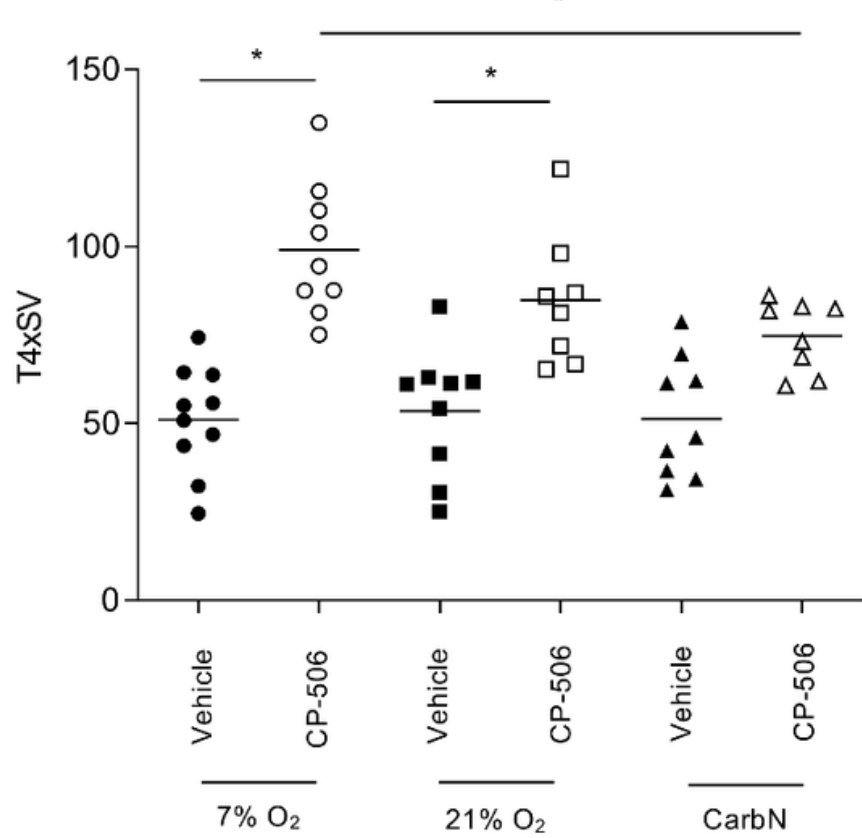

Figure 2

The antitumor effects of CP-506 are causally related to tumor oxygenation. (A) DMS114 tumors were exposed to vehicle or CP-506 (600 mg/kg, QD1), after which tumor hypoxia (pimonidazole) and DNA damage ( $\mathrm{\gamma H} 2 \mathrm{AX}$ ) were assessed and (B) quantified. (C) Excision assay of $\mathrm{H} 460$ tumors treated with radiation (10 Gy) and/or single dose CP-506 (900 or $1100 \mathrm{mg} / \mathrm{kg}$, IP). (D) Animals bearing MDA-468 tumor xenografts were exposed to modified breathing strategies (i.e. 7\% 02, 21\% 02, and carbogen (95\% $0 \neg 2,5 \% \mathrm{CO} 2)$ and nicotinamide (500 mg/kg) (CarbN)), during which CP-506 (600 mg/kg) was administered. (E) Ratios of HFpimo/HFEF5 of vehicle-treated tumors. The boxplot represents Q1, median, and Q3. Bars indicate the minimum and maximum values ( $n=6$ animals per treatment condition). $(F)$ 
Tumor growth was assessed and time to reach four times starting volume (T4xSV) was calculated. Data are presented as mean \pm SD.
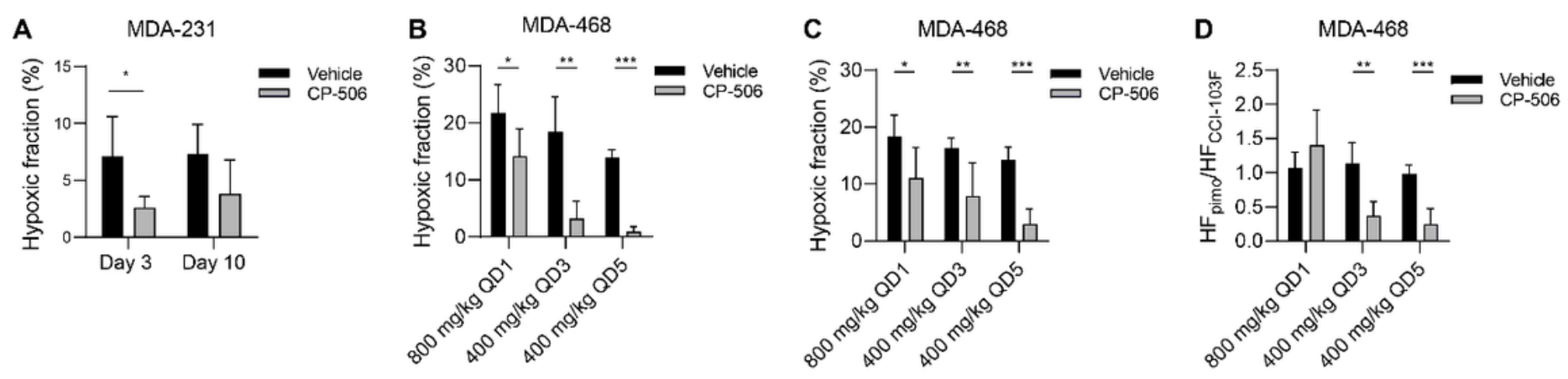

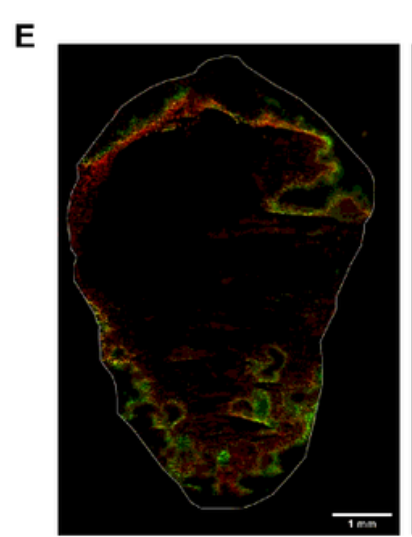

Vehicle

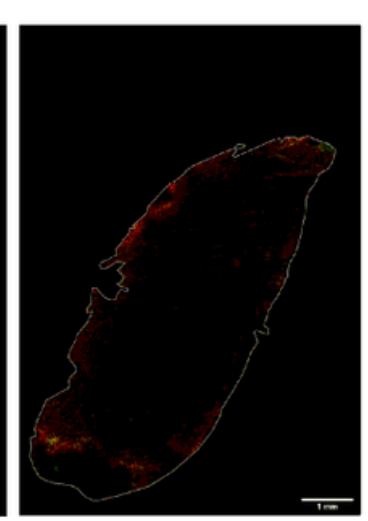

CP-506

MDA-468 (400 mg/kg, QD3)

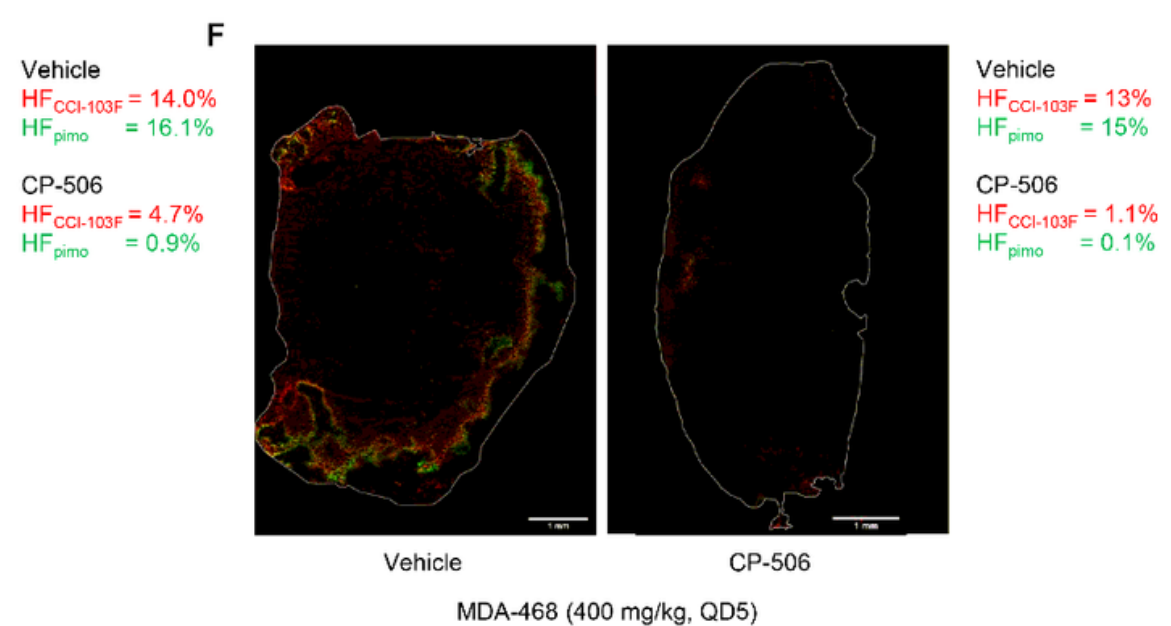

Figure 3

Impact of CP-506 on the hypoxic fraction in in vivo tumor xenografts. (A) Hypoxic fraction as assessed by pimonidazole positivity in MDA-231 tumor xenografts 3 days and 10 days after the end of CP-506 treatment $(800 \mathrm{mg} / \mathrm{kg}, \mathrm{QD} 5)$. Hypoxic fraction of MDA-468 tumors as assessed by pimonidazole (C) and $\mathrm{CCl}-103 \mathrm{~F}$ positivity at the end of different treatment schedules. (D) The ratio of HFpimo to HFCCI-103F after different treatment schedules. (E) Representative images of tumor hypoxia assessed by pimonidazole (green) and $\mathrm{CCl}-103 \mathrm{~F}$ (red) positivity in MDA-468 tumors (delineated in white) treated with vehicle or CP-506 (400 mg/kg, QD3) and (F) vehicle or CP-506 (400 mg/kg, QD5). Data are presented as mean \pm SD. 
A

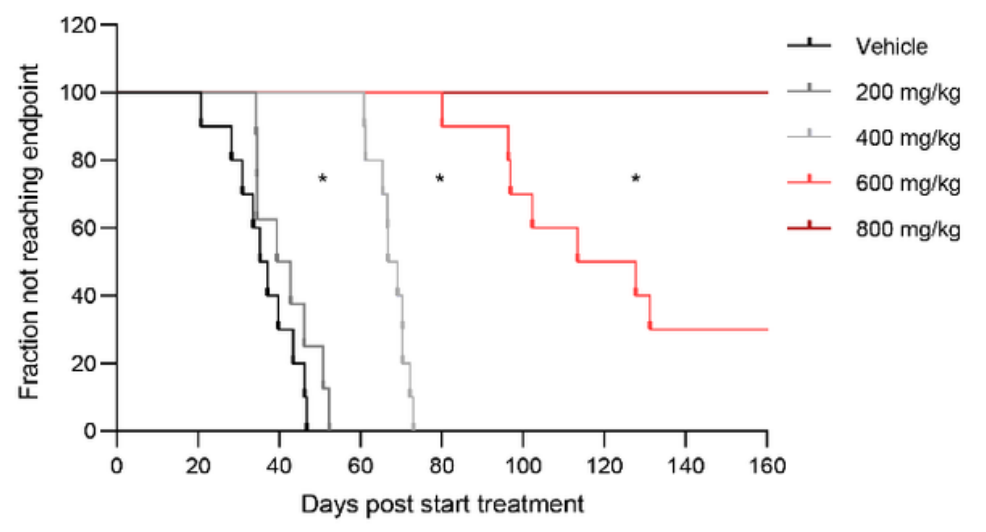

B
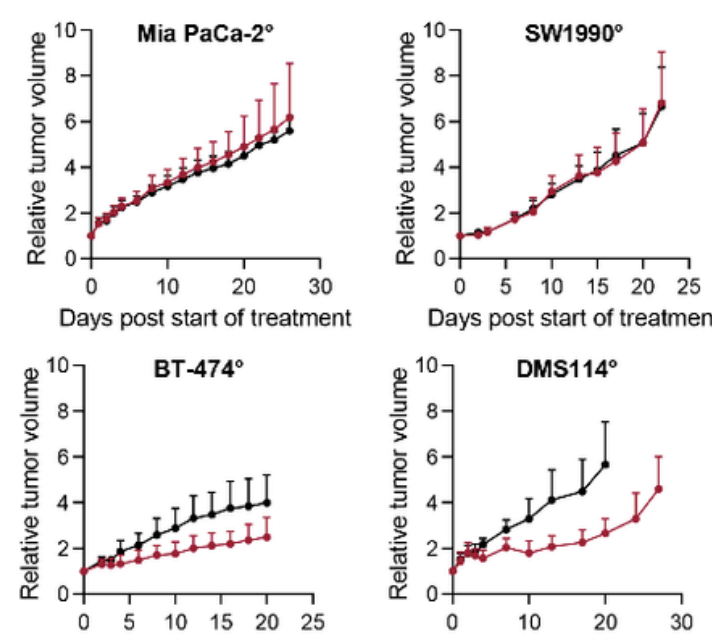

Days post start of treatment

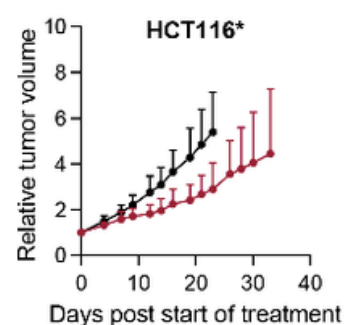

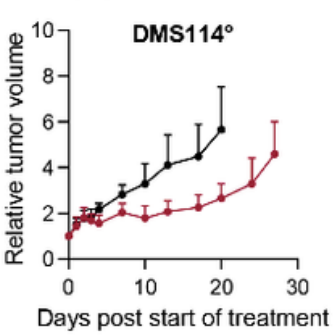

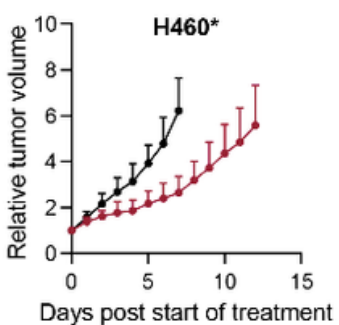

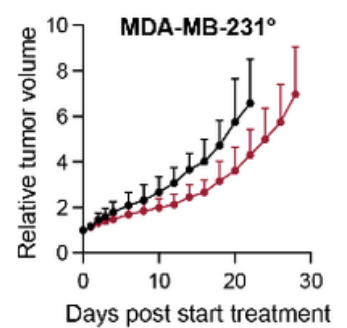

$\mathrm{H} 69^{\circ}$
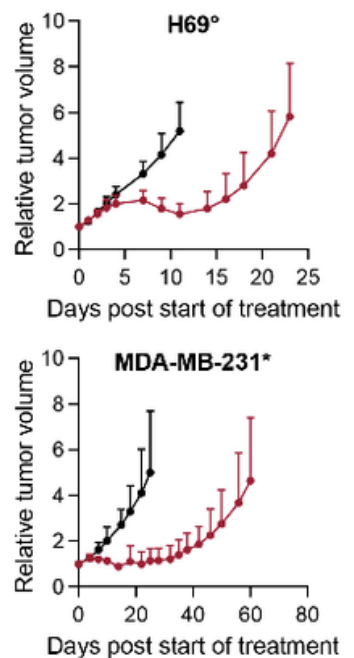
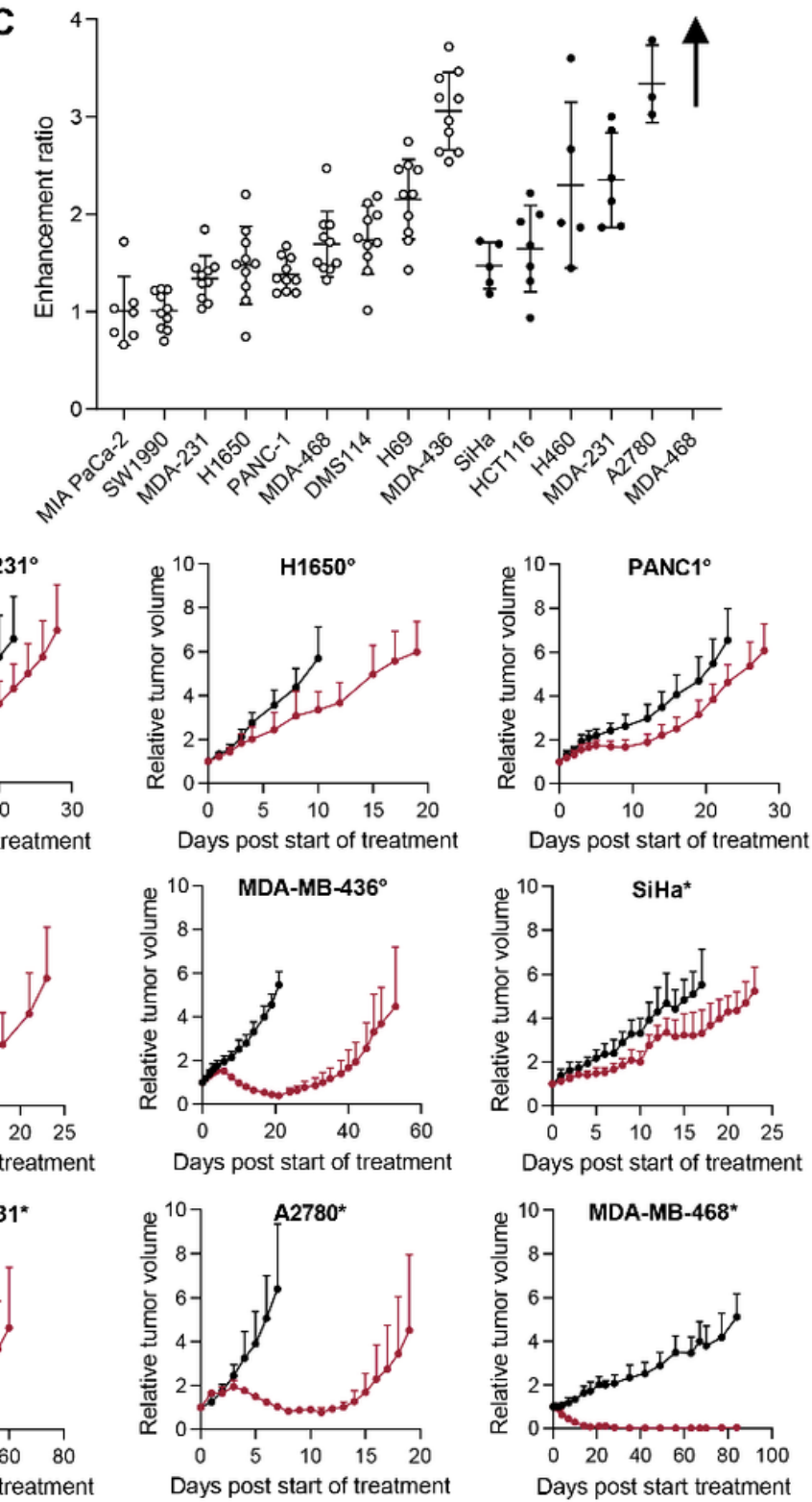

\section{Figure 4}

CP-506 inhibits tumor growth of several human xenografts. (A) Dose-dependence of the antitumor effect of CP-506 in MDA-468 tumor xenografts. Animals bearing MDA-468 xenografts were treated with increasing doses of CP-506 (200 - 800 mg/kg, IP, QD5). Tumor growth was assessed and Kaplan-Meier survival plots were constructed. Endpoint is defined as 2 times starting volume (SV). (B) CP-506 (600 (『) or $800\left(^{*}\right) \mathrm{mg} / \mathrm{kg}$, i.p.) was administered once per day for five days (QD5) in 15 different xenograft models. Tumor growth was monitored and tumor growth delay, defined as the time to reach four times start volume (T4xSV), was determined. (C) Enhancement ratios, defined as the ratio of T4xSV of CP-506treated mice to the average T4xSV of vehicle-treated mice was calculated. An upwards arrow indicated T4xSV is larger than 4. Data are presented as mean \pm SD.

\section{Supplementary Files}


This is a list of supplementary files associated with this preprint. Click to download.

- SupplementaryMaterialCP506aNovelHAP1.pdf 